\title{
Comparative genomics of downy mildews reveals potential adaptations to biotrophy
}

\author{
Kyle Fletcher', Steven J. Klosterman², Lida Derevnina ${ }^{1,5}$, Frank Martin², Lien D. Bertier', Steven Koike ${ }^{3,6}$ \\ Sebastian Reyes-Chin-Wo', Beiquan Mou ${ }^{2}$ and Richard Michelmore ${ }^{1,4^{*}}$
}

\begin{abstract}
Background: Spinach downy mildew caused by the oomycete Peronospora effusa is a significant burden on the expanding spinach production industry, especially for organic farms where synthetic fungicides cannot be deployed to control the pathogen. P. effusa is highly variable and 15 new races have been recognized in the past 30 years.

Results: We virulence phenotyped, sequenced, and assembled two isolates of $P$. effusa from the Salinas Valley, California, U.S.A. that were identified as race 13 and 14. These assemblies are high quality in comparison to assemblies of other downy mildews having low total scaffold count (784 \& 880), high contig $\mathrm{N}_{50} \mathrm{~S}(48 \mathrm{~kb} \& 52 \mathrm{~kb}$ ), high BUSCO completion and low BUSCO duplication scores and share many syntenic blocks with Phytophthora species. Comparative analysis of four downy mildew and three Phytophthora species revealed parallel absences of genes encoding conserved domains linked to transporters, pathogenesis, and carbohydrate activity in the biotrophic species. Downy mildews surveyed that have lost the ability to produce zoospores have a common loss of flagella/motor and calcium domain encoding genes. Our phylogenomic data support multiple origins of downy mildews from hemibiotrophic progenitors and suggest that common gene losses in these downy mildews may be of genes involved in the necrotrophic stages of Phytophthora spp.
\end{abstract}

Conclusions: We present a high-quality draft genome of Peronospora effusa that will serve as a reference for Peronospora spp. We identified several Pfam domains as under-represented in the downy mildews consistent with the loss of zoosporegenesis and necrotrophy. Phylogenomics provides further support for a polyphyletic origin of downy mildews.

Keywords: Peronospora effusa, Peronospora farinosa, Spinach downy mildew, Oomycete, Genomics, Peronospora lineage, Gene loss, Biotrophy

\section{Background}

Downy mildew diseases are caused by species of several genera of obligate biotrophic oomycetes and impact production of crops and ornamental plants worldwide [1]. The phylogenetic relationships of downy mildews to one another, as well as to closely related Phytophthora species (spp.), are unclear, with uncertainty as to how often the adaptation to obligate biotrophy has occurred [2-4]. Among the 19 downy mildew genera, Peronospora contains the highest number of species $(\sim 500)$ [1].

\footnotetext{
*Correspondence: rwmichelmore@ucdavis.edu

'The Genome Center, Genome and Biomedical Sciences Facility, University of California, 451 East Health Sciences Drive, Davis, CA 95616, USA

${ }^{4}$ Departments of Plant Sciences, Molecular \& Cellular Biology, Medical Microbiology \& Immunology, University of California, Davis 95616, USA Full list of author information is available at the end of the article
}

Peronospora spp. produce sexual oospores for survival in soil and plant debris, typical of oomycetes. However, unlike many oomycetes, the asexual sporangia of Peronospora germinate by forming a germ tube from the sporangia, rather than by releasing motile zoospores [1].

A prominent example of a destructive oomycete is Peronospora effusa, which causes spinach downy mildew; it is the most important pathogen of spinach globally $[1,5]$. Like other pathogens in this genus, $P$. effusa has a narrow host range, only infecting spinach (Spinacia oleracea) [6, 7]. This pathogen has occasionally been incorrectly grouped with other Peronospora spp., such as Peronospora schachtii (causal agent of chard downy mildew) under the umbrella Peronospora farinosa $[5,6,8-10]$, despite molecular data for distinct species $[11,12]$. A formal rejection of the

(c) The Author(s). 2018 Open Access This article is distributed under the terms of the Creative Commons Attribution 4.0 International License (http://creativecommons.org/licenses/by/4.0/), which permits unrestricted use, distribution, and reproduction in any medium, provided you give appropriate credit to the original author(s) and the source, provide a link to the Creative Commons license, and indicate if changes were made. The Creative Commons Public Domain Dedication waiver (http://creativecommons.org/publicdomain/zero/1.0/) applies to the data made available in this article, unless otherwise stated. 
name $P$. farinosa (a.k.a. Botrytis farinosa) was recently proposed, in part because $P$. farinosa could not be associated with a type specimen [13].

Demand for and production of fresh market spinach is consistently high in the United States of America [14] and control of downy mildew is essential for sustainable production of spinach, particularly on organic farms. While synthetic fungicides have been effective in managing downy mildew diseases in conventional production [15], such fungicides are unavailable for organic spinach production. Therefore, the introduction of genes for resistance to downy mildew into spinach through breeding currently provides the most effective option for disease control for the burgeoning organic industry [15]. Based upon reactions of cultivars in the differential set used for screening isolates, six major loci for resistance to $P$. effusa have been proposed $[8,9]$. Genome sequencing of $S$. oleracea identified 139 candidate resistance genes, five of which are closely linked to the $D M-1$ gene, which confers resistance to $P$. effusa race (R) 6 [16].

New virulent races of $P$. effusa have appeared rapidly after the deployment of cultivars with new resistance genes, leading to a large increase in the number of races designated over the past decade [5]. Prior to the 1990s, only three $P$. effusa races had been described [17], to date 17 races have been denominated based on reactions to differentially susceptible or resistant lines [5, 8, 9, 18, 19]. The reason for the rapid appearance of the new races of $P$. effusa is not understood; the recent finding of oospores of $P$. effusa in $\sim 16 \%$ of modern spinach seed lots has provided evidence for global movement of $P$. effusa on spinach seed [20] from seed production areas to fresh market production fields elsewhere. Sexual progeny from oospores will be highly variable because $P$. effusa is heterothallic $[10,21]$ and the global movement increases the potential for sexual recombination between novel combinations of isolates of $P$. effusa. While within-field genotypic diversity may be driven by asexual variation, the overall diversity of the species may be influenced by sexual recombination on broad temporal and geographic scales [19]. Oospores introduced into the fresh market production areas on spinach seed could introduce new combinations of virulence factors and contribute to the rapid demise of resistance genes.

Genome sequencing is now sufficiently inexpensive to permit the rapid sequencing and assembly of multiple isolates of small genome Peronospora spp. [22]. Recently the genomes of two Peronospora tabacina isolates were sequenced and shown to be compact and gene rich with fewer repeated sequences compared to other oomycetes [23]. While multiple species of several oomycete genera have been sequenced (e.g. Phytophthora, Pythium, Saprolegnia, Aphanomyces), only one genus (Plasmopara) of the obligately biotrophic downy mildews has had two species sequenced [3, 24, 25].

In this study, two isolates of $P$. effusa were collected from the field, virulence-phenotyped, and sequenced to produce high quality annotated assemblies comparable to other downy mildews and Phytophthora spp. P. effusa, like $P$. tabacina, has a small consensus genome size with few repeated sequences, although fewer gene models were identified. We contrasted these new assemblies and their gene models to other oomycetes and identified several domains that are under-represented in downy mildews with many orthologs missing when compared to Phytophthora spp. These data have implications to the loss of motile flagella and the necrotrophic mode of nutrition in downy mildews.

\section{Results}

Two isolates of $P$. effusa were collected from commercial spinach production fields in Monterey County, California in 2012 and 2013 and their virulence phenotypes tested by inoculations onto the standard differential set of resistant spinach cultivars to provide race designations as previously described [8]. Cultivars Avenger, Lion (Solomon), and Pigeon were resistant to the 2012 isolate indicating that it was race 13 (R13), while cultivars Califlay, Whale, and Lion were resistant to the 2013 isolate indicating that it was race 14 (R14).

An additional isolate was collected from Monterey County in January 2016 to determine total nuclear DNA content. Flow cytometry revealed three peaks indicating nuclear DNA contents of $80+/-2 \mathrm{Mb}, 149+/-9 \mathrm{Mb}$, and $300+/-18 \mathrm{Mb}$ (Additional file 1). These results are consistent with the $P$. effusa isolate surveyed containing populations of nuclei within a coenocytic mycelium; a small proportion is approximately $80 \mathrm{Mb}$, while the majority are $149 \mathrm{Mb}$. The larger $300 \mathrm{Mb}$ represents replicating $149 \mathrm{Mb}$ nuclei.

\section{Read processing and assembly}

Approximately 230 million 100 base-par (bp) paired-end reads were generated for both isolates that were reduced by $32 \%$ (R13) and 18\% (R14) after quality and k-mer trimming. Read filtering identified approximately 58 and 97 million read pairs associated with other oomycete assemblies and 65 and 77 million un-associated read pairs for R13 and R14, respectively. Optimal k-mer lengths for highest output statistics were empirically found to be between 91 and $99 \mathrm{nt}$ for both isolates. The top five BLASTn [26] filtered assemblies of R13 were assembled using k-mer sizes 92 to 96 and had scaffold $\mathrm{N}_{50}$ s ranging from $20.9 \mathrm{k}$-base-pair $(\mathrm{kb})$ to $23.8 \mathrm{~kb}$ with between 12 and 16 scaffolds over $100 \mathrm{~kb}$ with BUSCO [27] completeness (protist library) ranging from 94.9 to $97.9 \%$. The top five R14 assemblies (k-mer sizes 92-95+97) 
had scaffold $\mathrm{N}_{50}$ ranges of 18,157 to $18,478 \mathrm{bp}$, with 18 to 23 scaffolds over $100 \mathrm{~kb}$ and BUSCO completeness ranging from 95.3 to $97.4 \%$.

Post mitochondrial fragment filtering, pairwise merging of assemblies, from lowest scaffold count size to highest, and removal of fragments under $1 \mathrm{~kb}$, the scaffold $\mathrm{N}_{50}$ of isolates R13 and R14 rose to $52.8 \mathrm{~kb}$ and $42.8 \mathrm{~kb}$ respectively and the highest BUSCO [27] score previously observed was retained in both isolates, although the duplication rate had increased. A single round of redundancy removal with Redundans [28] and Haplomerger2 [29] resulted in the final assemblies, with little redundancy detected by BUSCO. Complete statistics for the intermediate assemblies generated are provided in Additional file 2.

P. effusa R13 was assembled into 1475 contigs and 785 scaffolds. The contig $\mathrm{N}_{50}$ was $48.369 \mathrm{~kb}$ and the scaffold $\mathrm{N}_{50}$ was $72.2 \mathrm{~kb}$. The assembly totaled $32.1 \mathrm{Mb}$, contained $0.26 \%$ gaps, and presented 68 scaffolds over $100 \mathrm{~kb}$, three of which were over $250 \mathrm{~kb}$. JELLYFISH [30] analysis produced an estimated haploid genome size of 44.1 Mega-base-pairs (Mb) with $26.6 \mathrm{Mb}$ inferred as single copy (Additional file 3). BUSCO [27] reported a completeness of $97.8,0.4 \%$ of complete BUSCOs were reported as duplicated and $2.2 \%$ reported as missing. There were no fragmented BUSCOs (Table 1).

P. effusa R14 was assembled in to 1275 contigs and 880 scaffolds. The contig $\mathrm{N}_{50}$ was $51.71 \mathrm{~kb}$ and the scaffold $\mathrm{N}_{50}$ was $61.4 \mathrm{~kb}$. The assembly totaled $30.8 \mathrm{Mb}$, contained $0.56 \%$ gaps and had 47 scaffolds over $100 \mathrm{~kb}$, one of which was over $250 \mathrm{~kb}$. JELLYFISH analysis produced an estimated haploid genome size of 41.2 Mb with $27.9 \mathrm{Mb}$ inferred as single copy (Additional file 3). BUSCO reported a completeness of $97.0 \%$, with no duplicated predictions and $0.4 \%$ fragmented predictions. The remaining $2.6 \%$ BUSCOs were reported as missing (Table 1).

\section{Assembly quality}

SyMap [31] plots showed that the two P. effusa assemblies had a high degree of collinearity although they are highly fragmented (Fig. 1). There were 237 and 214 scaffolds over $50 \mathrm{~kb}$ for $\mathrm{R} 13$ and R14, respectively; inter-isolate alignments were identified for $141 \mathrm{R} 13$ and 134 R14 of these scaffolds. When compared to P. sojae, $177 \mathrm{R} 13$ and $167 \mathrm{R} 14$ scaffolds over $50 \mathrm{~kb}$ could be aligned with $23 P$. sojae scaffolds over $50 \mathrm{~kb}$.

\section{Repeat masking and LTR analysis}

Repeat libraries for both isolates were generated using RepeatModeler [32], identifying 98 elements in R13 and 88 in R14. In both isolates most elements identified were Long Terminal Repeat (LTR) Retrotransposons (Additional file 4). The LTR profiles of the two isolates of $P$. effusa were similar to one another, although the density profile indicates that isolate R14 may have more similar Copia elements than R13 (Additional file 5).

\section{Annotation}

Initial SNAP [33] hidden Markov model (HMM) training with MAKER [34] resulted in 7853 gene predictions for R13. Subsequent runs with intermediate SNAP bootstrapping produced predictions ranging from 6493 to 9601 models, the mean residue length ranged from 407 to 482 amino acids. Equivalent bootstrapping of the same HMM run on P. effusa R14 produced predictions ranging from 6691 to 9034 gene models, the mean residue length ranged from 407 to 476 amino acids (Additional file 6). Further assessment of each individual unfiltered run showed mean BLAST [26] scores back to the training data ranging between 569 to 671 for R13 and 603 to 678 for R14 (Additional file 6). When orthology analysis was performed for each run against the training data, the number of orthogroups detected varied from 4803 to 7117 for R13 and 5363 to 7073 for R14 (Additional file 6). The mean e-value for Pfam [35] domains, scoring under $1 \mathrm{e}^{-5}$, of individual MAKER runs ranged from $1.99 \mathrm{e}^{-7}$ to $2.20 \mathrm{e}^{-7}$ for $\mathrm{R} 13$ and $1.99 \mathrm{e}^{-7}$ to $2.23 \mathrm{e}^{-7}$ for R14 (Additional file 6). For R13 the MAKER run with the highest mean BLAST score, second highest orthogroup count, and lowest mean Pfam e-value was considered the best. For R14, the best annotation set scored the highest mean BLAST score, highest orthogroup count, and lowest mean Pfam e-value (Additional file 6). Investigating gene models at unique loci from alternative MAKER runs did not produce a high scoring set of gene models; therefore, integrating unique models from multiple runs was not performed.

Independent translation of the entire genome of both isolates, followed by effector identification through a combination of HMM-profiling and string searches (Additional file 7) resulted in a total of 148 and 137 putative single open reading frame (ORF) effectors. Intersecting these loci with MAKER predictions identified 41 and 43 previously unpredicted loci for each isolate (Additional file 8). Putative effectors were manually curated in cases of overlapping or flanking ORFs. Reconciliation of these cases resulted in three multi-exonic effectors for both isolates, in every case the first exon contained a signal peptide, RxLR motif, and EER motif as well as possibly more than one WY domain. The second exon always contained at least one WY domain. These were added with the other ORF predictions to their respective annotated gene sets (Additional file 9). The effector prediction pipeline was run on the final gene sets, producing counts of 113 and 107 putative 


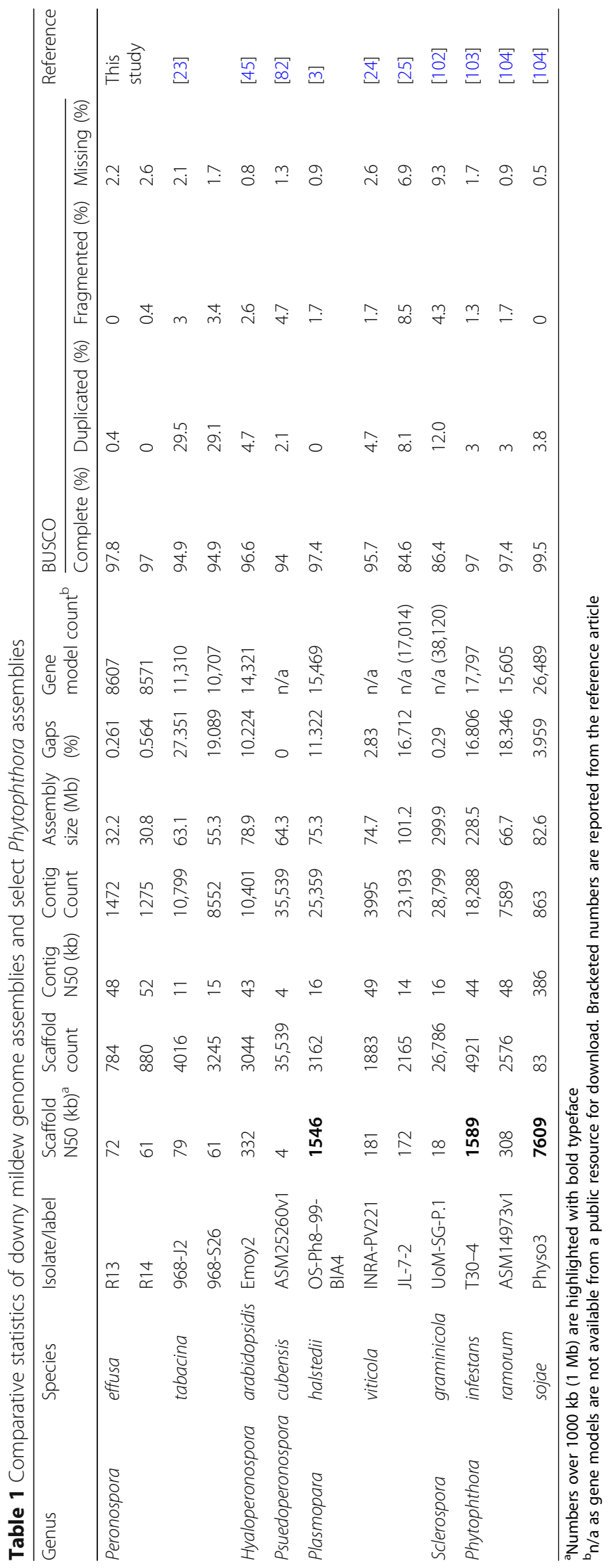




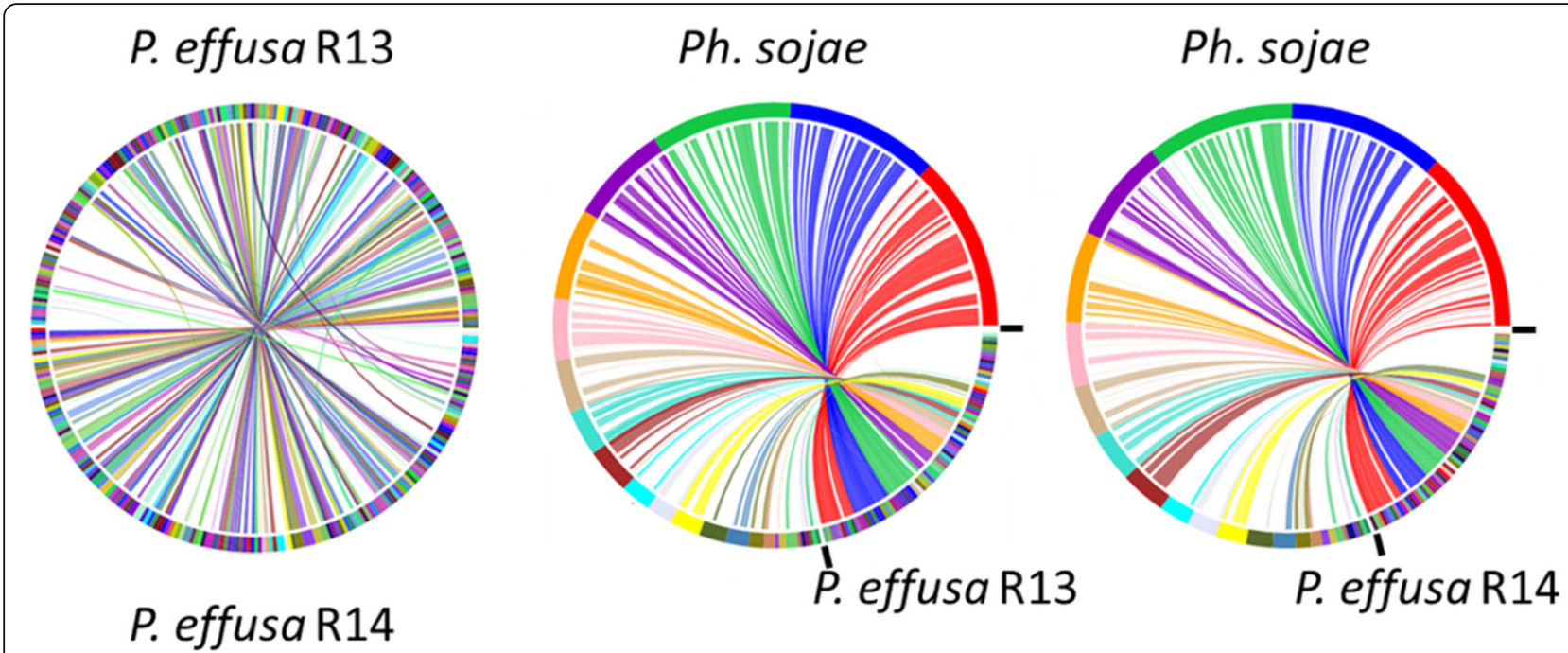

Fig. 1 SyMap plots of $P$. effusa R13 aligned against P. effusa R14, P. sojae v3.0 against $P$. effusa R13 and P. sojae v3.0 against $P$. effusa R14. The plots aligned against $P$. sojae are scaled so the area of the plot occupied by $P$. sojae scaffolds is scaled to the size of the $P$. effusa sequences. No scaling is applied to the $P$. effusa cross isolate comparison

effectors for the draft assemblies of R13 and R14 respectively (Table 2).

\section{Comparative analysis}

Gene models encoding putative pathogenicity domains were identified through InterProScan for each assembly (Table 3). T-tests indicated that the frequencies of gene models encoding several of these domain types were significantly different between Phytophthora spp. and downy mildews, as was the total frequency of pathogenicity related models. The frequency of pathogenicity-associated gene models in Phytophthora spp. ranged between 0.026 to 0.036, while all downy mildews had lower incidences ranging from 0.013 to 0.017 . The two isolates of $P$. effusa had the highest incidence of pathogenicity genes of the downy mildews analyzed.

Table 2 Putative effectors identified through regular expression and HMMs

\begin{tabular}{lll}
\hline Category & R13 & R14 \\
\hline RxLR-[DE][DE][ER] & 33 & 34 \\
[GHQ]xLR-[DE][DE][ER] & 7 & 7 \\
RxL[GKQ]-[DE][DE][ER] & 14 & 12 \\
RxLR-WY & 12 & 12 \\
[GHQ]xLR-WY & 0 & 0 \\
RxL[GKQ]-WY & 1 & 0 \\
RxLR-[DE][DE][ER]-WY & 24 & 20 \\
[GHQ]xLR-[DE][DE][ER]-WY & 1 & 0 \\
RxL[GKQ]-[DE][DE][ER]-WY & 1 & 2 \\
Total & 93 & 87 \\
CRN (Secreted) & $20(8)$ & $20(5)$ \\
\hline
\end{tabular}

Analysis (chi-squared) of Pfam domains revealed 96 as significantly enriched or depleted relative to their expected distributions, scoring below the Bonferroni adjusted e-value of $1.33 \mathrm{e}^{-5}$ (Additional file 10) in at least one of the four multi-species comparisons performed (Table 4). Of these, six were enriched but were excluded because they had a skewed over-representation in P. tabacina (possibly due to under assembly of the genomes; see section on K-mer analysis and heterozygosity below) compared to P. effusa and Hyaloperonospora arabidopsidis; excluding P. tabacina resulted in insignificant scores for these six. The 90 remaining Pfam domains were all indicated as depleted in the Peronospora lineage made up of $P$. effusa, $P$. tabacina and $H$. arabidopsidis, when compared to Phytophthora spp. (Table 4). In addition, 64 of the 90 domains were also under-represented in Plasmopara halstedii including 24 of which could be grouped as phytopathology, transporter and carbohydrate associated domains. When $P$. halstedii, the only downy mildew analyzed that has motile flagella, was grouped with the Phytophthora spp., 26 domains obtained a more significant score including 14 in the classes: flagella apparatus and calcium associated domains. These five classes contained $42 \% \quad(38 / 90)$ of the domains detected as significantly depleted; the other $58 \%$ could not be assigned to one of these classes (Additional file 10).

Orthology analysis between the two isolates of $P$. effusa identified 7314 overlapping orthogroups, of which 6833 were single copy. Nine orthogroups, containing 54 genes and a further 653 singletons unassigned to orthogroups were found exclusive to R13, while 10 
Table 3 Putative pathogenicity domain encoding genes of $P$. effusa and related oomycetes

\begin{tabular}{|c|c|c|c|c|c|c|c|c|c|c|c|}
\hline & \multicolumn{4}{|c|}{ Peronospora } & \multirow{3}{*}{$\begin{array}{l}\text { Hyaloperonospora } \\
\text { arabidopsidis }\end{array}$} & \multirow{3}{*}{$\begin{array}{l}\text { Plasmopara } \\
\text { halstedii }\end{array}$} & \multirow{2}{*}{\multicolumn{3}{|c|}{ Phytophthora }} & \multirow{3}{*}{$\begin{array}{l}\text { Phytophthora } \\
\text { spp. vs. } \\
\text { downy } \\
\text { mildews } \\
\text { t-test }\end{array}$} & \multirow{3}{*}{$\begin{array}{l}\text { Peronospora } \\
\text { lineage vs. } \\
\text { Phytophthora } \\
\text { spp. }+P \text {. } \\
\text { halstedii } \\
\text { t-test }\end{array}$} \\
\hline & \multicolumn{2}{|l|}{ effusa } & \multicolumn{2}{|c|}{ tabacina } & & & & & & & \\
\hline & $\mathrm{R} 13$ & R14 & $\overline{J 2}$ & S26 & & & sojae & infestans & ramorum & & \\
\hline Serine protease & 14 & 13 & 21 & 12 & 12 & 27 & 42 & 37 & 36 & & \\
\hline Aspartic protease & 11 & 9 & 17 & 7 & 15 & 26 & 147 & 20 & 116 & $<0.05$ & \\
\hline Cysteine protease & 16 & 17 & 18 & 17 & 16 & 20 & 28 & 27 & 30 & & \\
\hline Metalloprotease & 16 & 18 & 14 & 16 & 25 & 17 & 38 & 24 & 26 & & \\
\hline $\begin{array}{l}\text { Kazal-like serine } \\
\text { protease inhibitor }\end{array}$ & 1 & 1 & 2 & 2 & 7 & 17 & 45 & 35 & 17 & $<0.01$ & $<0.001$ \\
\hline $\begin{array}{l}\text { Cystatin-like cysteine } \\
\text { protease inhibitor }\end{array}$ & 1 & 1 & 1 & 1 & 1 & 3 & 4 & 6 & 4 & $<0.05$ & $<0.01$ \\
\hline Cutinase & 0 & 0 & 0 & 0 & 2 & 2 & 15 & 4 & 4 & $<0.01$ & $<0.05$ \\
\hline Pectin lyase & 11 & 13 & 6 & 8 & 22 & 19 & 122 & 100 & 82 & $<0.001$ & $<0.05$ \\
\hline CAP domain & 39 & 42 & 45 & 48 & 38 & 72 & 155 & 112 & 104 & $<0.01$ & \\
\hline NPP1-like & 9 & 10 & 17 & 14 & 21 & 19 & 80 & 28 & 62 & $<0.05$ & $<0.05$ \\
\hline Elicitin-like & 16 & 15 & 12 & 8 & 20 & 20 & 77 & 54 & 61 & $<0.001$ & $<0.05$ \\
\hline Jacalin & 10 & 10 & 7 & 7 & 10 & 20 & 31 & 22 & 27 & & $<0.05$ \\
\hline Frequency & 0.0168 & 0.0173 & 0.0141 & 0.0130 & 0.0132 & 0.0167 & 0.0295 & 0.0262 & 0.0361 & $<0.001$ & $<0.05$ \\
\hline
\end{tabular}

orthogroups, containing 70 genes and a further 638 singletons were exclusive to R14. These isolate-specific genes represented $8 \%$ of the total predicted genes (Fig. 2a). Reads were mapped between isolates to determine the inter isolate coverage of coding regions and to determine if SNPs/short-indels could be detected in genes to determine why the proteins were inferred as absent. Mapping reads of R14 to R13 revealed 40 R13 genes that had low coverage, one of which encoded an RxLR effector. Seven genes contained indels within their coding regions. Additionally, 28 genes, including one encoding a necrosis inducing protein (NPP1), had SNPs which introduced a premature stop codon. SNPs in 14 genes resulted in the loss of their start codon and the loss of the stop codon in 22 genes, relative to the genes predicted in $\mathrm{R} 13$. When $\mathrm{R} 13$ reads were mapped to $\mathrm{R} 14$, ten genes had low coverage, including two RxLR effectors, which had a single residue difference between one another. Fifteen genes, including one RxLR contained indels within their coding regions. Additionally, 31 genes, including one CRN, had SNPs which introduced premature stop codons, 22 genes, including one RxLR and a gene encoding a protein kinase domain, had SNPs which caused the loss of the stop codon and nine lost their start codon, relative to the genes predicted for R14. These proteins, especially those with putative virulence/ necrotic function may be useful as diagnostic markers for each race (Additional file 11). Repeating the analysis on the two previously sequenced isolates of $P$. tabacina [23] revealed 6818 overlapping orthogroups, 3869 of which were single copy and 19\% of the gene models reported as unique to either of the isolates (Fig. 2b).

Species-level orthology analysis were performed by combining the gene models predicted for both isolates of each Peronospora spp. and comparing these with predictions from single isolates of $H$. arabidopsidis, $P$. halstedii, Phytophthora infestans, Phytophthora sojae and Phytophthora ramorum. This identified 12,835 orthogroups (Fig. 2c). Of these, 4893 were ubiquitous to all species, 2865 were present in at least two Phytophthora spp. and exclusive to this clade, 956 orthogroups were ubiquitous and exclusive to Phytophthora spp. and $P$. halstedii, 237 were ubiquitous to all assemblies except $P$. halstedii, 395 were ubiquitous to all assemblies except $H$. arabidopsidis, and 112 excluded both Peronospora spp. (Fig. 2c). Only 43 orthogroups, containing 154 gene models were unique to Peronospora spp. (Additional file 12). $P$. effusa scored its highest pairwise orthology coefficient with $P$. tabacina (0.82) followed by with $H$. arabidopsidis (0.77), P. halstedii (0.68) and Phytophthora spp. (0.54 to 0.58). The orthology coefficient showed a general trend grouping Phytophthora spp. (0.72 to 0.86) and Peronospora spp. plus $H$. arabidopsidis (0.76-0.82); however, $P$. halstedii did not group well with either, scoring 0.65 to 0.67 with Phytophthora spp. and 0.66 to 0.68 with Peronospora spp./ H. arabidopsidis. P. effusa shared more orthologous groups, than P. tabacina or H. arabidopsidis, with all Phytophthora spp. and P. halstedii and consistently scored a higher orthology coefficient than P. tabacina and H. arabidopsidis, when compared to all three Phytophthora spp. 
Table 4 Chi-square results of Pfam domain representation in gene models of 9 oomycete assemblies

\begin{tabular}{|c|c|c|c|c|c|c|}
\hline Pfam & P. effusa vs all & Peronospora spp. vs. All & $\begin{array}{l}\text { Peronospora } \\
\text { lineage vs. all }\end{array}$ & $\begin{array}{l}\text { Downy mildew vs. } \\
\text { Phytophthora spp. }\end{array}$ & Pfam title & Category \\
\hline PF11051 & & $8.34 \mathrm{e}^{-10}$ & $6.11 \mathrm{e}^{-10}$ & $8.62 e^{-10}$ & Mannosyltransferase & Carbohydrate associated \\
\hline PF00232 & & & & $7.81 e^{-8}$ & Glycosyl hydrolase family 1 & \\
\hline PF00295 & & $3.66 \mathrm{e}^{-6}$ & $7.04 e^{-8}$ & $4.71 e^{-11}$ & Glycoside hydrolase family 28 & \\
\hline PF00933 & & $1.20 \mathrm{e}^{-5}$ & $1.70 \mathrm{e}^{-6}$ & $1.69 e^{-8}$ & $\begin{array}{l}\text { Glycosyl hydrolase family } 3 \mathrm{~N} \text { terminal } \\
\text { domain }\end{array}$ & \\
\hline PF01762 & & & & $3.93 e^{-6}$ & Galactosyltransferase & \\
\hline PF01915 & & & $3.19 e^{-6}$ & $2.45 e^{-8}$ & Glycoside hydrolase family 3 & \\
\hline PF00612 & & $2.17 e^{-6}$ & $2.88 \mathrm{e}^{-7}$ & & IQ calmodulin binding motif & Calcium associated \\
\hline PF13202 & & $4.68 \mathrm{e}^{-6}$ & $9.71 \mathrm{e}^{-9}$ & $9.92 e^{-7}$ & EF hand & \\
\hline PF13499 & & $3.70 \mathrm{e}^{-8}$ & $4.59 e^{-10}$ & $9.75 e^{-8}$ & EF hand & \\
\hline PF13833 & & & $1.13 \mathrm{e}^{-7}$ & & EF hand & \\
\hline PF00225 & & $1.16 \mathrm{e}^{-7}$ & $7.33 e^{-10}$ & $2.75 e^{-6}$ & Kinesin motor domain & Flagella / Motor associated \\
\hline PF07728 & & $9.14 e^{-6}$ & $6.35 \mathrm{e}^{-8}$ & & $\begin{array}{l}\text { ATPases associated; dynein related } \\
\text { subfamily }\end{array}$ & \\
\hline PF03028 & & $6.28 e^{-9}$ & $4.15 e^{-12}$ & $9.94 e^{-8}$ & $\begin{array}{l}\text { Dynein heavy chain and region D6 of } \\
\text { dynein motor }\end{array}$ & \\
\hline PF08385 & & & $2.85 \mathrm{e}^{-6}$ & & Dynein heavy chain & \\
\hline PF08393 & & $1.94 e^{-9}$ & $1.51 \mathrm{e}^{-13}$ & $3.36 \mathrm{e}^{-9}$ & Dynein heavy chain, $\mathrm{N}$-terminal region 2 & \\
\hline PF12774 & & $8.43 e^{-9}$ & $1.23 \mathrm{e}^{-12}$ & $4.65 e^{-8}$ & $\begin{array}{l}\text { ATPases associated; P-loop containing } \\
\text { dynein motor region }\end{array}$ & \\
\hline PF12775 & & $2.04 e^{-8}$ & $4.35 \mathrm{e}^{-12}$ & $2.18 e^{-7}$ & $\begin{array}{l}\text { ATPases associated; P-loop containing } \\
\text { dynein motor region }\end{array}$ & \\
\hline PF12777 & & $3.49 e^{-9}$ & $3.50 \mathrm{e}^{-13}$ & $9.69 e^{-9}$ & Microtubule-binding stalk of dynein motor & \\
\hline PF12780 & & $2.21 e^{-7}$ & $6.99 \mathrm{e}^{-11}$ & $1.49 e^{-6}$ & $\begin{array}{l}\text { ATPases associated; P-loop containing } \\
\text { dynein motor region-D4 }\end{array}$ & \\
\hline PF12781 & & $2.74 e^{-8}$ & $6.61 \mathrm{e}^{-12}$ & $3.62 e^{-7}$ & $\begin{array}{l}\text { ATPases associated; P-loop containing } \\
\text { dynein motor region-D5 }\end{array}$ & \\
\hline PF00050 & & $1.46 e^{-8}$ & $2.47 e^{-9}$ & $1.06 e^{-7}$ & Kazal-type serine protease & Phytopathology associated \\
\hline PF00734 & & & $4.92 \mathrm{e}^{-6}$ & $1.14 \mathrm{e}^{-5}$ & Fungal cellulose binding domain & \\
\hline PF07648 & & $9.31 e^{-8}$ & $4.11 \mathrm{e}^{-10}$ & $2.77 e^{-08}$ & Kazal domain & \\
\hline PF00544 & & $5.44 e^{-7}$ & $1.88 \mathrm{e}^{-6}$ & $9.83 e^{-10}$ & Pectate lyase & \\
\hline PF00964 & & $1.17 e^{-8}$ & $5.92 e^{-10}$ & $1.87 e^{-12}$ & Elicitin & \\
\hline PF02902 & & $2.59 e^{-10}$ & $1.53 \mathrm{e}^{-14}$ & $1.62 \mathrm{e}^{-20}$ & $\begin{array}{l}\text { ULP1 protease family, C-terminal catalytic } \\
\text { domain }\end{array}$ & \\
\hline PF03211 & & $3.68 \mathrm{e}^{-13}$ & $1.21 \mathrm{e}^{-13}$ & $6.77 e^{-19}$ & Pectate lyase & \\
\hline PF05630 & & $3.81 e^{-09}$ & $3.88 e^{-9}$ & $9.46 e^{-12}$ & Necrosis inducing protein & \\
\hline PF09461 & & & & $5.22 e^{-6}$ & Phytotoxin PCF & \\
\hline PF16810 & $6.38 \mathrm{e}^{-10}$ & $4.13 \mathrm{e}^{-32}$ & $6.61 e^{-45}$ & $1.08 \mathrm{e}^{-64}$ & RxLR phytopathogen effector protein. & \\
\hline PF00083 & & $3 e^{-7}$ & $1 e^{-10}$ & $3 e^{-6}$ & Sugar (and other) transporter & Transporter associated \\
\hline PF00520 & $1.03 e^{-6}$ & $2.86 \mathrm{e}^{-14}$ & $3.15 \mathrm{e}^{-21}$ & $7.97 e^{-21}$ & Ion channel family & \\
\hline PF00005 & $3.84 \mathrm{e}^{-6}$ & $1.32 \mathrm{e}^{-19}$ & $9.28 e^{-23}$ & $5.70 e^{-32}$ & $A B C$ transporter & \\
\hline PF00664 & & $2.11 e^{-9}$ & $8.42 \mathrm{e}-11$ & $2.34 \mathrm{e}-14$ & $\begin{array}{l}\text { Transmembrane domain of } A B C \\
\text { transporters }\end{array}$ & \\
\hline PF01061 & & $2.36 \mathrm{e}^{-17}$ & $2.11 e-20$ & $3.45 e-29$ & ATP-binding cassette transporter & \\
\hline PF03083 & & $1.63 e^{-7}$ & $1.88 \mathrm{e}-10$ & $2.16 \mathrm{e}-14$ & $\begin{array}{l}\text { Sugar efflux transporter for intercellular } \\
\text { exchange }\end{array}$ & \\
\hline PF03092 & & & & $5.98 \mathrm{e}^{-7}$ & BT1 family & \\
\hline PF06422 & & $3.41 e^{-10}$ & $5.84 \mathrm{e}-10$ & $1.37 e-13$ & CDR ABC transporter & \\
\hline
\end{tabular}

The bold score for each Pfam domain is the best $p$-value obtained from all four comparisons 
A

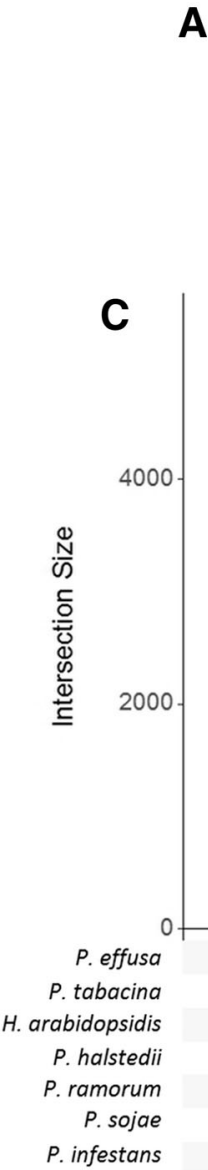

P. effusa R13

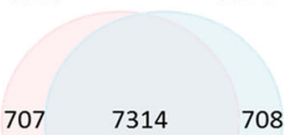

\section{B P. tabacina}

$\mathrm{J} 2$

S26

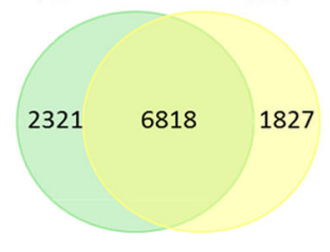

\begin{tabular}{|c|c|}
\hline Species & $\begin{array}{c}\text { Unique gene } \\
\text { model count }\end{array}$ \\
\hline P. effusa & 1807 \\
P. tabacina & 3095 \\
H. arabidopsidis & 4951 \\
P. halstedii & 5332 \\
P. ramorum & 1062 \\
P. sojae & 6064 \\
P. infestans & 2543 \\
\hline
\end{tabular}

\begin{tabular}{ll}
$1145 \quad 1126$ \\
\hline
\end{tabular}
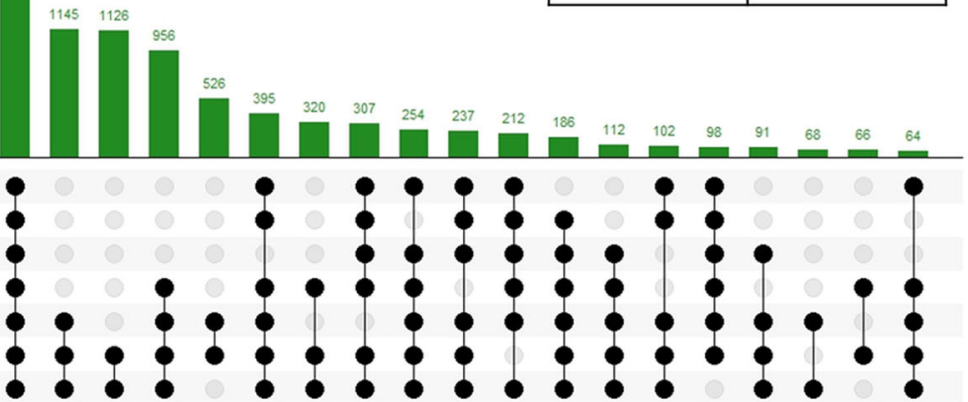

Fig. 2 Orthology analysis of oomycete gene models. a Venn diagram depicting the number of orthogroups shared between two $P$. effusa isolates and unique gene counts. $\mathbf{b}$ Venn diagram depicting the number of orthogroups shared between two $P$. tabacina and unique gene counts. c UpsetR plot demonstrating the number of orthogroups shared by sets of oomycete species. The intersection size is the number of orthogroups and the black dots on the $x$-axis represent whether the orthogroups are present or absent in that set. For instance, the first bar demonstrates that 4893 orthogroups are shared among all oomycetes, while the second demonstrated that 1145 are shared among

Phytophthora spp. and are absent in the downy mildews. Only intersections over 60 orthogroups are depicted. The table inset shows how many gene models are unique to each species and include models not assigned to orthogroups and models included in orthogroups made up from a single species

and $P$. halstedii (Table 5). These higher orthology scores for $P$. effusa are consistent with high quality of the gene model predictions for this species.

There were 709 orthogroups containing at least one protein encoding a Pfam domain inferred as under-represented in Peronospora species/H. arabidopsidis and grouped under one of the previously defined categories (Table 4). These were visualized to investigate the absence of the orthologs in downy mildew species (Fig. 3). While a core component of each orthogroup, typically the high gene number groups in all categories except phytopathogenicity, are retained across all oomycetes tested (Table 6), the majority of orthogroups for each category have missing orthologs for Peronospora sp. and $H$. arabidopsidis. When these are grouped with $P$. halstedii over $50 \%$ of orthogroups have detectable orthologs for downy mildew species in every category except phytopathogenicity, with a much larger fraction of motor and calcium associated domain encoding orthologs being identified than carbohydrate and transporter orthologs in P. halstedii (Table 6). The counts and IDs of the proteins contained within each orthogroup are supplied as Additional file 13.

Orthologous groups associated with nitrogen and sulphur assimilation were investigated (Table 7). Neither of the two nitrate reductase paralogs present in all three Phytophthora spp. were detected in the six assemblies of the four downy mildew species analyzed. The nitrate transporter orthogroup contained 4 to 6 paralogs for each Phytophthora spp. with 1 to 2 homologs identified for each Peronospora spp./H. arabidopsidis; no ortholog was identified from $P$. halstedii. Sulfite reductase genes 
Table 5 Pairwise overlaps of orthology groups (top right) and calculated orthology coefficients (bottom left bold and bracketed on intersecting diagonal) of publicly available gene models of downy mildew genome assemblies and select Phytophthora spp.

\begin{tabular}{|c|c|c|c|c|c|c|c|}
\hline & P. effusa $a^{a}$ & P. tabacina ${ }^{a}$ & H. arabidopsidis & P. halstedii & P. ramorum & P. sojae & P. infestan \\
\hline P. effusa ${ }^{a}$ & $7430(1)$ & 6646 & 6425 & 6572 & 6557 & 6860 & 6882 \\
\hline P. tabacina ${ }^{\text {a }}$ & 0.82 & 7209 (1) & 6283 & 6421 & 6378 & 6680 & 6755 \\
\hline H. arabidopsidis & 0.77 & 0.76 & 7211 (1) & 6388 & 6365 & 6638 & 6668 \\
\hline P. halstedii & 0.68 & 0.67 & 0.66 & $8578(1)$ & 7469 & 8020 & 8055 \\
\hline P. ramorum & 0.58 & 0.57 & 0.56 & 0.65 & 9963 (1) & 9436 & 9016 \\
\hline P. sojae & 0.54 & 0.52 & 0.52 & 0.64 & 0.76 & 11,798 (1) & 10,711 \\
\hline P. infestans & 0.57 & 0.56 & 0.55 & 0.67 & 0.72 & 0.86 & 11,272 (1) \\
\hline
\end{tabular}

${ }^{a}$ Two isolates used in analysis

were identified in one isolate of P. tabacina and both $P$. effusa assemblies but were not detected in $H$. arabidopsidis and $P$. halstedii. Orthologs of other nitrogen and sulphur assimilation associated enzymes were nearly ubiquitous in the tested assemblies, except no orthologs of glutamate synthase were detected in one of the isolates of P. tabacina and no orthologs of glutamine synthetase were detected in P. halstedii.

\section{K-mer analysis and heterozygosity}

KAT density plots [36] were made to investigate heterozygosity in P. effusa. Two clusters of 21-mers are visible in both plots in addition to the many low-frequency 21-mers due to sequencing errors and contaminants (Fig. 4a). R14 has strong homozygous k-mer signal and a weak heterozygous signal at half coverage. R13 has strong homozygous k-mer signal and a significant signal at higher than half coverage. The same analysis of two $P$. tabacina isolates [23] detected strong homozygous k-mer signal and two heterozygous signals at half and quarter coverage consistent with the presence of multiple distinct haplotypes due to polyploidy, heterokaryosis, or mixtures for both isolates (Fig. 4a).

Spectra-cn plots that are also generated by KAT [36] were used to investigate the frequencies of k-mers in common between the read sets and assemblies of $P$. effusa. R14 contains one significant peak with the majority of 21-mers being represented once in the assembly, consistent with a high-quality assembly of a predominantly homozygous organism (Fig. 4b). The majority of 21-mers of the R13 read set were represented once, regardless of the k-mer frequency in the read set; this indicates that neither cluster of k-mers in R13 was heterozygous because most of the $\mathrm{k}$-mers in the lower coverage peak were incorporated in the assembly rather than the anticipated proportion (50\%) of the k-mers being absent. Therefore, the assembly of R13 seems to be of high quality but these k-mer plots are not consistent with a simple diploid genome. In contrast, spectra-cn plots of $P$. tabacina confirmed that both isolates contained three clusters of k-mers with a high proportion of k-mers from the first peaks absent in the assembly, approximately half the k-mers absent from the second peaks, and a small fraction absent from the homozygous peaks. Both isolates of $P$. tabacina had high levels of k-mer duplication; a significant proportion in the homozygous peak was represented twice as much as expected in the assembly indicating that both P. tabacina assemblies are under-assembled (Fig. 4b).

Reads were mapped back to the respective assemblies to identify single nucleotide polymorphisms (SNPs) and investigate the frequency of reads supporting the alternative allele in $P$. effusa. In R13, 106,714 heterozygous SNP sites were identified and 74,690 in R14, indicating 0.33 and $0.24 \%$ heterozygosity for R13 and R14, respectively (Fig. 4c). Plots of the frequency of reads supporting the alternative allele at each SNP revealed a clear peak at 0.5 in both isolates as expected in a diploid; however, a smaller second peak was present for both isolates at $\sim 0.33$. When only SNPs in genes were considered, the SNP count was reduced to 31,041 and 17,943 inferring a 0.23 and $0.13 \%$ heterozygosity in the predicted gene space of R13 and R14, respectively. Plots of the frequency of reads supporting the alternative allele of genic SNPs retained the peak at 0.5 , although the peak at 0.33 was greatly reduced, containing $~ 5.7 \%$ (R13) to $8.6 \%$ (R14) of the genic SNPs (Fig. 4c). Collectively, these data indicate that the allele frequencies of numerous SNPs in $P$. effusa are not consistent with the 1:1 ratio expected in a diploid organism.

The normalized coverage of each predicted gene in R13 was more variable than in R14 (Fig. 4d). While the majority of the $\sim 8600$ predicted genes had around 1 to $1.2 \mathrm{x}$ normalized coverage in both isolates, 851 gene models in R13 had 0.6 to $0.8 x$ coverage; in contrast, only 67 genes in $\mathrm{R} 14$ had 0.6 to $0.8 \mathrm{x}$ coverage. There was no significant deviation in representation of the 513 Pfam domains encoded by these genes.

\section{Phylogenetics}

A maximum likelihood tree with 1000 bootstraps was produced from 49 concatenated, single copy genes predicted by BUSCO [27] from both $P$. effusa isolates and all 


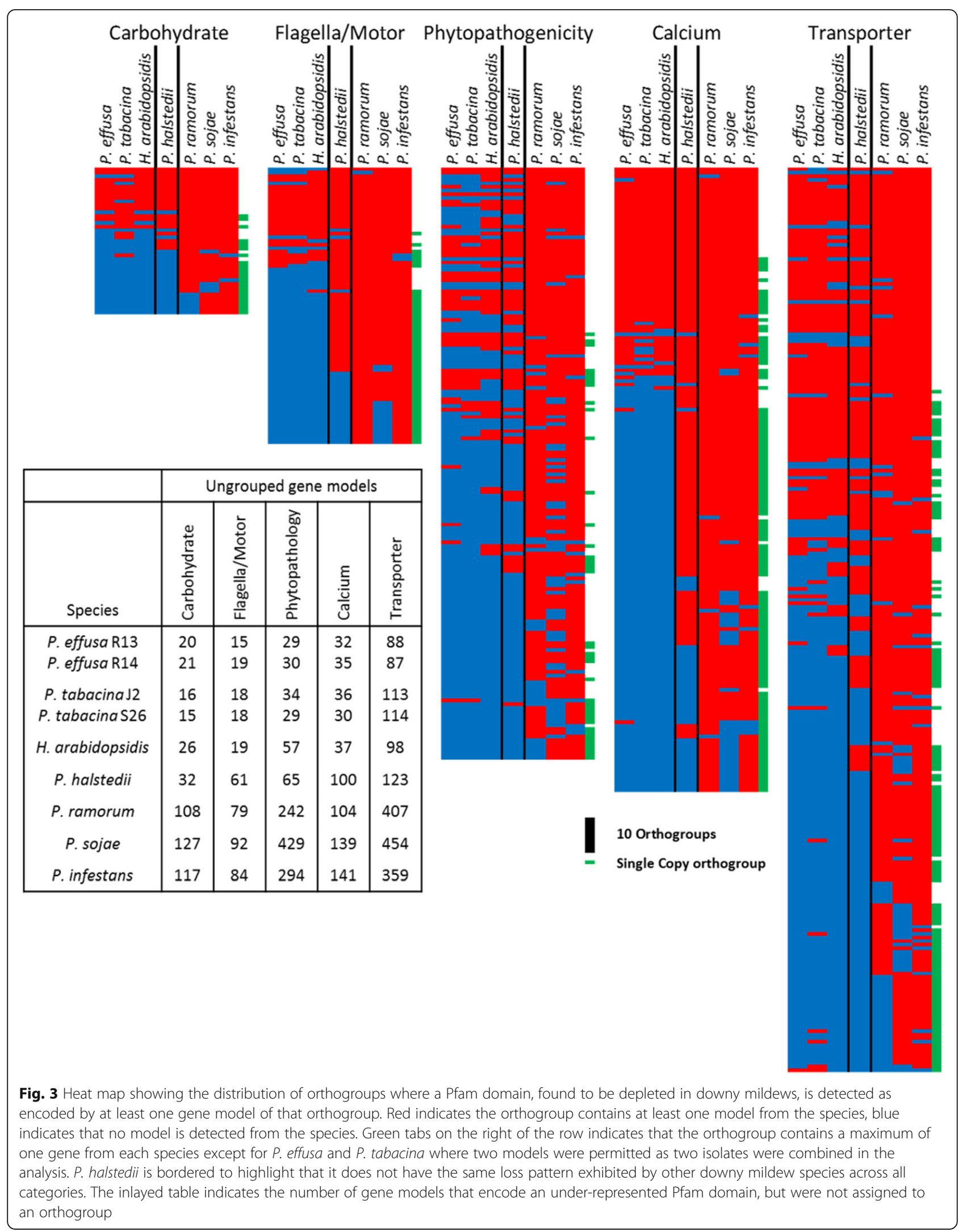




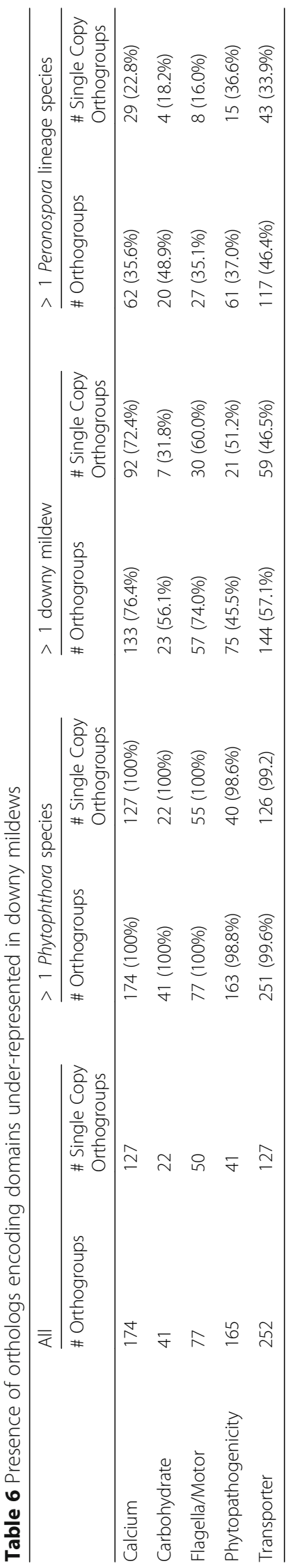




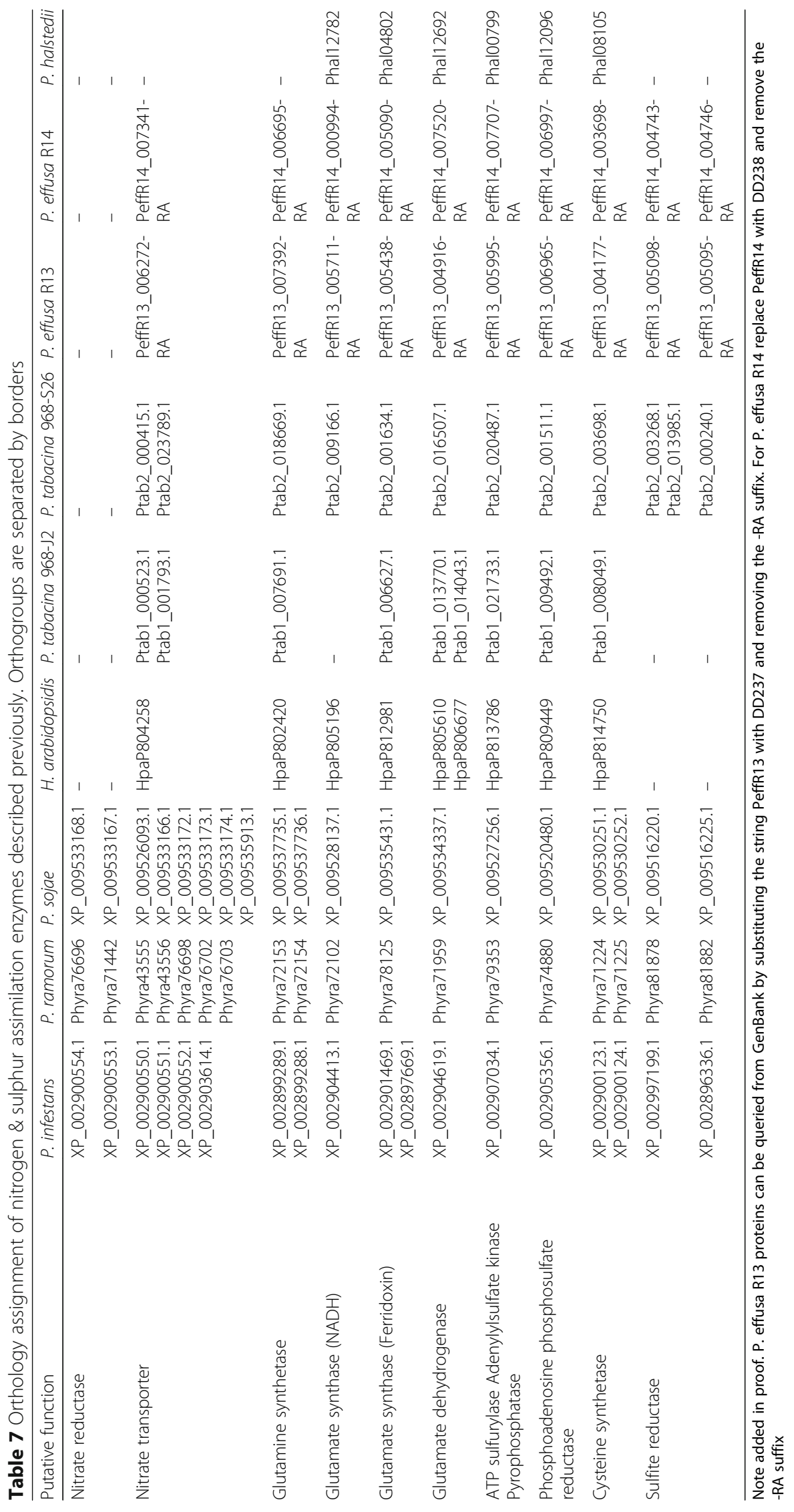




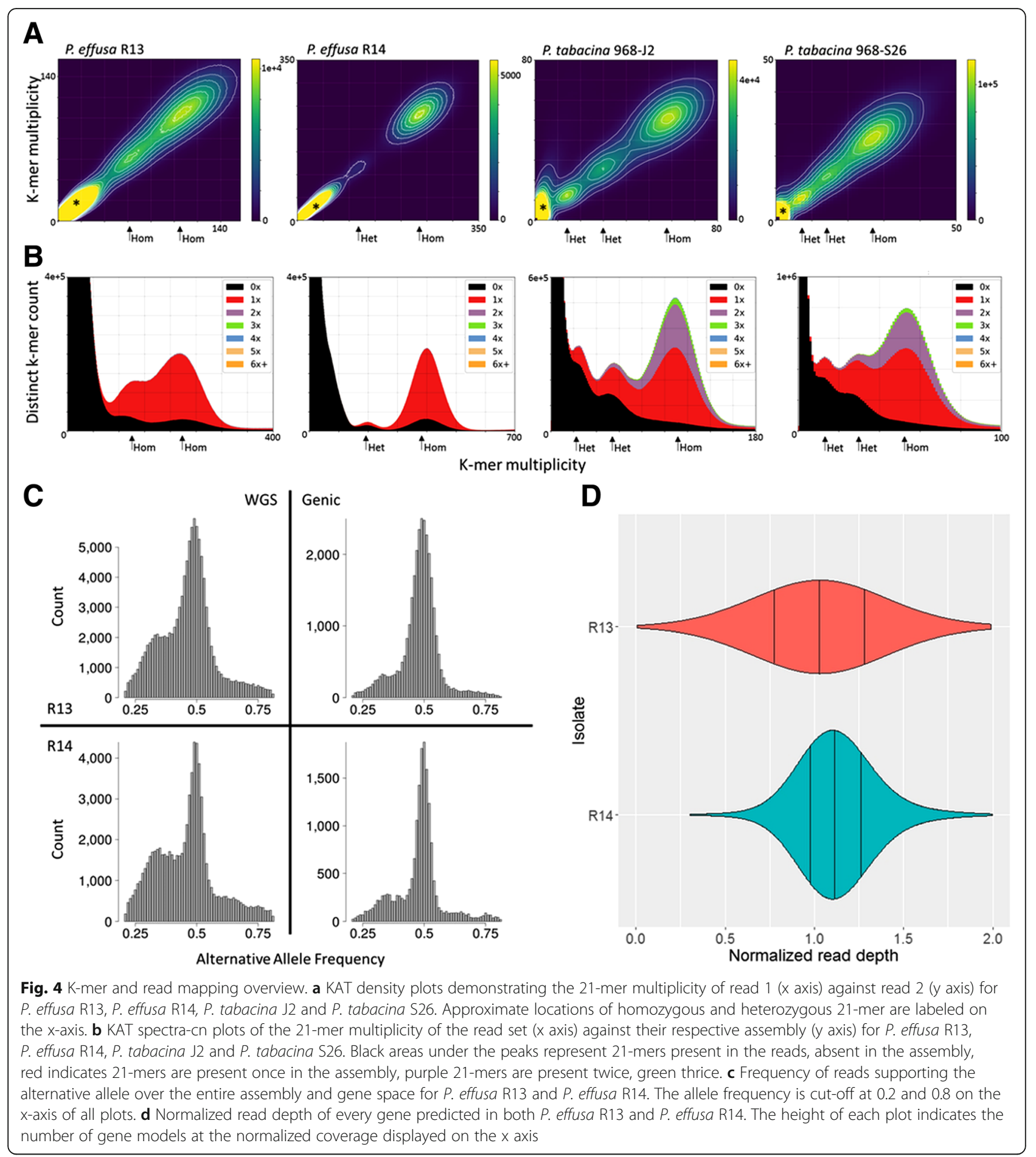

published downy mildew assemblies available from NCBI, plus three diverse Phytophthora spp. and rooted with Pythium ultimum as the out-group (Fig. 5). Two downy mildew clades were evident. $P$. effusa was in the larger clade with $P$. tabacina and clusters with Pseudoperonospora cubensis, $H$. arabidopsidis and Sclerospora graminicola. The second downy mildew clade is made up of the three isolates of two Plasmopara spp., which were more closely related to $P$. infestans than to the other downy mildews.

\section{Mitochondria}

The mitochondrial genomes of race 13 and 14 isolates were both 41,318 bp in size with a GC content of $22.8 \%$ (GenBank accessions MH142315 and MH325167, 


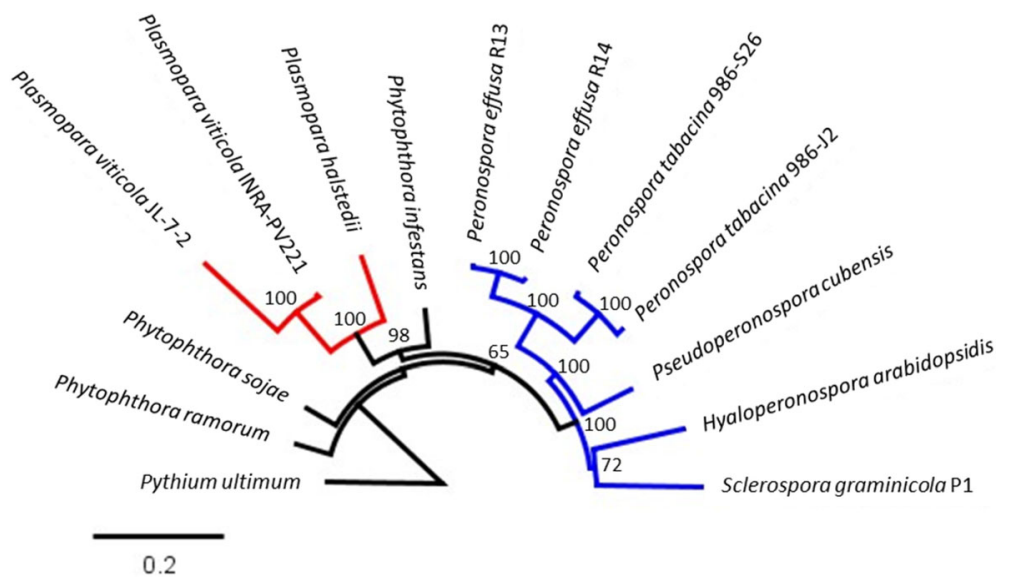

Fig. 5 Maximum likelihood phylogeny of 49 BUSCO gene models, identified as conserved as a single copy gene across all assemblies surveyed. Node labels indicate the score after 1000 bootstraps. The scale bar indicates the nucleotide divergence per site

respectively). The coverage of the mitochondrial assemblies was 2081 and 1003x for R13 and R14, respectively. Sequences of both genomes were identical and had the same organization as P. tabacina (KT893455) with the exception of an inverted repeat (IR) that was present in P. effusa. Coding regions constituted $93.9 \%$ of the genome with $13.3 \%$ of this total representing hypothetical coding regions. A total of 35 known genes (encoding 18 respiratory chain proteins, 16 ribosomal proteins, and an import protein, ymf16 of the secY-independent pathway), the $r n l$ and $r n s$, and 25 tRNA genes encoding for 19 amino acids were present. In addition, there were five hypothetical proteins (ymf $98, y m f 99, y m f 100, y m f 101$ and orf32) in common with other oomycete mitochondrial genomes [23, 37-42] and five putative ORFs that were unique to P. effusa. Four of the unique ORFs (orf 181, orf131, orf201 and orf277) were located between the rns and $\operatorname{cox} 2$ genes (in the same location as the unique putative ORFs of $P$. tabacina but encoded in the opposite orientation) and another between the atp1 and nad5 genes (orf209). BLAST queries to GenBank identified no significant sequence similarities for orf181, orf201, and orf209; however, there was moderate sequence similarity of orf131 and orf 277 with putative ORFs in mitochondrial genomes of $P$. tabacina and Phytophthora sojae (DQ832717). In orf131 of P. effusa, bases 287 to 393 were $75 \%$ identical to the $5^{\prime}$ end of orf $269 a$ of $P$. tabacina, while bases 7 to 262 were $69 \%$ identical to the $3^{\prime}$ end of this putative ORF. The 3 ' end of $P$. tabacina orf 269a (bases 323 to 743) shared similarity with putative ORFs from $P$. sojae with $85 \%$ identity to the spacer and $5^{\prime}$ end of orf116, while bases 59 to 320 are $89 \%$ identical to part of orf101. Bases 8-677 of $P$. effusa orf 277 were $73 \%$ identical to all but the last $100 \mathrm{bp}$ of $P$. tabacina orf $269 \mathrm{~b}$ and bases $8-785$ sharing $70 \%$ identity with the $5^{\prime}$ end of P. tabacina orf 290 .
A feature of the $P$. effusa mitochondrial genome that was not present in P. tabacina was the presence of an IR. The first arm of the IR was located between orf 201 and orf 277 with $382 \mathrm{bp}$ of the $5^{\prime}$ end representing the $5^{\prime}$ end of orf201. The second arm of the IR was located between atp 1 and orf 209 with $382 \mathrm{bp}$ of the $5^{\prime}$ end of the IR the $5^{\prime}$ end of orf209. The sequence for both arms of the IR is not a perfect match because there is a $2 \mathrm{bp}$ deletion after base 667 in the first arm relative to the second repeat, hence the sizes are $872 \mathrm{bp}$ and $874 \mathrm{bp}$, respectively. In addition to the IR there was also a $47 \mathrm{bp}$ repeat present in two head to tail copies between orf277 and $\operatorname{cox} 2$.

\section{Discussion}

We present here two highly contiguous genome assemblies of an oomycete downy mildew pathogen. These assemblies of $P$. effusa contain 70 to $75 \%$ of the haploid genome size that is estimated to be 41 to $44 \mathrm{Mb}$ by k-mer analysis. The missing component probably encodes repeat sequences that were not resolved from paired-end reads of short insert fragments, as the assemblies exceed the estimated size $(\sim 27 \mathrm{Mb})$ of the single copy portion of the genome. This assembly size is approximately half the smallest peak detected by flow cytometry and a quarter of the predominant peak, consistent with $P$. effusa being composed of a population of diploid and polyploid nuclei, as previously described for Phytophthora spp. [43, 44]. In comparison to assemblies of other downy mildews, these assemblies of $P$. effusa have fewer scaffolds and fewer contigs and have high BUSCO percent completeness scores indicating that they contain most of the gene space. The contig $\mathrm{N}_{50}$ of the $P$. effusa assemblies are similar to those of other downy mildews; however, the incorporation of long distance information from different technologies 
into assemblies of $H$. arabidopsidis [45] and Plasmopara spp. [3, 24, 25] resulted in higher scaffold $\mathrm{N}_{50}$ s. The scaffold $\mathrm{N}_{50} \mathrm{~s}$ are similar to those reported for $P$. tabacina, which were assembled using mate-pair reads [23] (Table 1).

The scaffolds of $P$. effusa are highly syntenic with those of P. sojae v3.0 (Fig. 1). P. sojae v3.0 is the most contiguous oomycete assembly available with 83 scaffolds and $99.12 \%$ of $82.6 \mathrm{Mb}$ assembled into 31 scaffolds over $50 \mathrm{~kb}$. The assemblies of $P$. effusa are more fragmented; $69 \%$ of R13 was encoded in 237 scaffolds over $50 \mathrm{~kb}$, while $60 \%$ of R14 was contained in 214 scaffolds over 50 kb. The SyMap [31] plots (Fig. 1) provided little evidence for chimeric scaffolds in $P$. effusa. Furthermore, 177 R13 scaffolds and 167 R14 scaffolds could be ordered against $23 P$. sojae scaffolds possibly indicative of pseudomolecules; pseudomolecules were not generated because of the potential for genome re-arrangements reported within the Peronosporales [46-50]. The generation of improved assemblies with chromosomal pseudomolecules awaits the future application of technologies that utilize long distance information [51].

The repertoires of annotated genes differed between $P$. effusa and $P$. tabacina. The assemblies of $P$. tabacina have a high BUSCO [27] duplication scores ( 30\%), indicating that these may not be high-quality consensus assemblies (i.e. the assembly is not representative of a single haplotype) and may have inflated the estimated number of gene models in $P$. tabacina compared to $P$. effusa. The BUSCO score of P. tabacina indicates that $\sim 30 \%$ of the $\sim 11,000$ gene models may be duplicated; this is supported by the duplication of single copy regions in these assemblies detected by KAT [36] plots (Fig. 4b). If this is the case, there are $\sim 8000$ gene models in $P$. tabacina, similar to $P$. effusa. Interestingly, 4148 gene models were unique to one or the other isolate of $P$. tabacina (Fig. 2b) and 3095 genes were unique to $P$. tabacina when compared to other oomycetes (Fig. 2). The BUSCO duplication score for $P$. effusa was less than $0.5 \% ; 1807$ genes were unique to $P$. effus $a$ when compared to other oomycetes (Fig. 2). Therefore, these isolates of P. tabacina may have more dispensable genes or not all genes were successfully assembled and annotated in both isolates of P. tabacina. This difference could be due to the isolates of $P$. effusa being genetically more similar to one another than the two isolates of $P$. tabacina, therefore having less opportunity to differentiate their gene repertoire. This difference in diversity is supported by both isolates of $P$. effusa having identical mitochondrial sequences, while those of the two P. tabacina isolates differed by seven SNPs, three indels and copy number of a 128 bp repeat [23]. Other oomycetes also have a higher unique gene content compared to P. effusa (Fig. 2) as well as good BUSCO scores, possibly indicating specialized sets of genes or misannotations in the other species. Our analysis was based on annotation developed using the transcriptomes of other oomycetes including Hyaloperonospora, Bremia and Phytophthora species [52-55]. RNAseq data for $P$. effusa may increase the number of unique or specialized genes identified in this species. Further genome and transcriptome sequencing of more isolates are required to characterize the pan genomes and extent of specialization in multiple oomycete species.

The effector repertoire of $P$. effusa is reduced in comparison to Phytophthora spp., similar to other downy mildews [3, 23, 45]. P. effusa has fewer RxLR motif encoding effectors, $(\sim 90)$ than $P$. tabacina $(\sim 165)$, although our analysis had the additional requirement of a degenerate EER motif or WY domain being encoded, which was not used for the predictions of $P$. tabacina [23]. More Crinklers (CRNs) were reported for P. tabacina ( 130) than P. effusa (20; Table 2). P. effusa had a marginally higher incidence of genes models encoding putative pathogenicity associated domains but not RxLRs or CRNs (Table 3); $1.7 \%$ of the gene models of each isolate encoded a putative pathogenicity domain vs. $\sim 1.4 \%$ of the gene models for P. tabacina and $\mathrm{H}$. arabidopsidis. P. effusa has a similar frequency of pathogenicity associated genes as $P$. halstedii, while over $2.5 \%$ of Phytophthora spp. gene models encode putative pathogenicity domains. Less than $3 \%$ of the predicted genes in P. effusa are implicated in pathogenicity.

Several Pfam [35] domains were found to be depleted in downy mildews, possibly reflecting adaptations to biotrophy (Table 6; Fig. 3). Eight transporter-associated Pfam domains were significantly depleted in $P$. effusa and all other downy mildews compared to Phytophthora spp. (Table 4) similar to previous reports [23, 45]. Many orthogroups containing gene models encoding these domains were not detected in downy mildews (Fig. 3, Table 6). The same analysis revealed the absence of orthogroups containing genes encoding carbohydrate binding or pathogenicity domains from all downy mildews (Fig. 3, Table 6). These observations are consistent with observations of the reduction in the frequency of pathogenicity associated proteins (Table 3 ) encoding kazal domains, pectate lyase, elicitin, and necrosis inducing proteins. Therefore, the repertoire of pathogenicity proteins seems to be consistently reduced in the biotrophic downy mildews.

Three of the four downy mildew species also had a reduction in calcium binding and flagella associated domains (Fig. 3, Table 6). The absence of flagella-associated domains is expected because $P$. halstedii is the only downy mildew species analyzed which produces zoospores $[1,49,56]$. The absence of genes encoding calcium associated domains (Fig. 3, Table 6) is consistent with the upregulation of one of the depleted domains 
(EF-hand) during sporangial development and cleavage during zoospore formation [55]. Genes encoding these domains would be under reduced selection in the absence of flagella. The missing genes encoding calcium-associated domains are good candidates for genes associated with zoospore biology.

Phylogenomics resolved two independent lineages of downy mildews (Fig. 5), consistent with other studies $[2,3]$, but not with those that infer a single origin [4]. Our topology placed Peronospora spp. in a clade that included $H$. arabidopsidis, P. cubensis and S. graminicola. This clade was separate from a clade that included the two species of Plasmopara spp. analyzed. The consistent loss of genes encoding transporter, phytopathogenicity, and carbohydrate-associated domains between these clades indicates that these genes are functional in the necrotrophic stages of Phytophthora spp. [57] and therefore have been lost in the biotrophic downy mildews.

The allele frequencies of $P$. effusa were not consistent with those of a regular diploid (Fig. 4c). Both isolates had alternative allele frequencies of $\sim 0.33$ in addition to the peak at 0.5 expected for a diploid. The absence of heterozygous 21-mers in P. effusa R14 (Fig. 4a) and the low frequency of SNPs detected when reads were mapped back to the assembly indicated that this isolate was largely homozygous. The frequency of SNPs was slightly higher in R13 though this was not proportional to the two clusters of 21-mers. Additionally, the 21-mers were not at the expected coverage for heterozygous loci (Fig. 4a). The majority of 21-mers from both of these clusters were present in the assembly (Fig. 4b), although the assembly size of R13 was not inflated relative to R14. If this was heterozygosity in R13 then half the 21-mers would be expected to be absent [36]. These results implied that few of the 21-mers were heterozygous in R13. The 21-mer profile of R13 cannot be explained by a mixture of isolates; shared 21-mers in a mixture of two isolates would be present in the highest coverage cluster and the lower coverage cluster would be made up of 21-mers differentiating the two. This is not what was observed (Fig. 4b). In addition, these 21-mers should not all be present in an assembly of a mixture of isolates because they would produce bubbles in the assembly graph that would be collapsed in a consensus assembly. The 21-mer profile of R13 indicates that this isolate has an ambiguous genomic architecture, in which a proportion of its genome has either been lost or duplicated. This was supported by the normalized read depth of genes (Fig. 4d), in which R13 had a wider spectrum of normalized read coverage than R14. While measurements by flow cytometry were not possible for these two isolates, later measurements of a separate isolate revealed that some nuclei were smaller at $80 \mathrm{Mb}$ than the majority of $2 \mathrm{C}$ nuclei at $149 \mathrm{Mb}$ (Additional file 1). The basis of the apparent variation in nuclear DNA size warrants further investigation to determine the prevalence of aneuploidy and polyploidy in $P$. effusa as has previously been documented for Phytophthora spp. [43, 44, 46, 58].

The mitochondrial genome of $P$. effusa is circular in orientation, approximately $41.3 \mathrm{~kb}$ in size and exhibited no sequence divergence between the genomes of race 13 and 14. The size is similar to other oomycetes (Table 8). The mitochondrial genome of $P$. effusa encodes the same common suite of genes, including the putative ORFs ymf16, ymf98, ymf99, ymf100, and ymf101 as observed in the related taxa. There were also an additional four putative ORFs of unknown function encoded between rns and cox 2 and one putative ORF between the atp 1 and nad5 genes that are unique to P. effusa, two of which share some level of sequence identity with a putative ORF in P. tabacina and P. sojae. The locations of the four species-specific putative ORFs between rns and cox 2 were the same as in P. tabacina and P. cubensis. Inverted repeats (IRs) have been observed in the mitochondrial genomes of the Peronosporomycete Pythium and Saprolegniomycetes Saprolegnia, Achyla, Thraustotheca and Aphanomyces, but these typically represent between 34 to $73 \%$ of the genome and encode the large and small ribosomal RNAs [37-39, 41, 59, 60]. While an IR is present in P. effusa, it is atypical for an oomycete because it is less than $900 \mathrm{bp}$ and does not encode rRNA. These features are similar to the $1150 \mathrm{bp}$ inverted repeat present in P. ramorum [40]. Recombination between the small IRs in P. ramorum generated isomers of the genome where the region between the repeats was also present in an inverted orientation [61] additional experimentation is needed with $P$. effusa to confirm if this is occurring in this taxon as well.

\section{Conclusions}

We sequenced, assembled and annotated two isolates representing distinct races of $P$. effusa, the causal oomycete of spinach downy mildew. These assemblies are high quality (Table 1) and will serve as good references for this genus of over 500 species [1]. Approximately 8600 gene models were identified in each isolate that shared a high level of orthology between one another and with other oomycetes. Genes encoding domains associated with pathogenicity, transporters, and carbohydrate-binding were depleted across multiple downy mildews compared to Phytophthora spp. indicative of a parallel gene loss during the evolution of obligate biotrophy and genes associated with flagella were consistently absent in the non-flagellate downy mildews. These isolates of $P$. effusa were predominantly homozygous. High quality annotated assemblies of more isolates are required to resolve the complex genome architecture of $P$. effusa. 
Table 8 Mitochondrial assembly statistics across the oomycetes

\begin{tabular}{|c|c|c|c|c|c|c|}
\hline Family & Genus species & Isolate & Accession & Length & Inverted Repeat? & Inverted repeat length \\
\hline \multirow[t]{18}{*}{ Peronosporaceae } & \multirow[t]{2}{*}{ Peronospora effusa } & R13 & & $41.3 \mathrm{~kb}$ & $\mathrm{Y}$ & $0.87 \mathrm{~kb}$ \\
\hline & & R14 & & $41.3 \mathrm{~kb}$ & Y & $0.87 \mathrm{~kb}$ \\
\hline & \multirow[t]{2}{*}{ P. tabacina } & $968-J 2$ & NC028331 & $43 \mathrm{~kb}$ & N & \\
\hline & & $968-\$ 26$ & KT893456 & $43 \mathrm{~kb}$ & N & \\
\hline & \multicolumn{2}{|l|}{ Psuedoperonospora cubensis } & KT072718 & $38.6 \mathrm{~kb}$ & N & \\
\hline & Phytophthora andina & EC3425 & HM590419 & $37.9 \mathrm{~kb}$ & N & \\
\hline & \multirow[t]{4}{*}{ P. infestans } & 80029 & AY894835 & $37.9 \mathrm{~kb}$ & $N$ & \\
\hline & & $15 / 99$ & AY898627 & $39.8 \mathrm{~kb}$ & N & \\
\hline & & $94-52$ & AY898628 & $39.8 \mathrm{~kb}$ & $N$ & \\
\hline & & W4 & NC002387 & $38 \mathrm{~kb}$ & N & \\
\hline & P. ipomoeae & PIC99167 & HM590420 & $37.9 \mathrm{~kb}$ & $\mathrm{~N}$ & \\
\hline & P. mirabilis & PIC99114 & HM590421 & $37.8 \mathrm{~kb}$ & N & \\
\hline & P. nicotianae & & KY851301 & $37.6 \mathrm{~kb}$ & $\mathrm{~N}$ & \\
\hline & P. phaseoli & P18 & HM590418 & $37.9 \mathrm{~kb}$ & N & \\
\hline & P. polonica & & KT946598 & $40.5 \mathrm{~kb}$ & $\mathrm{~N}$ & \\
\hline & \multirow[t]{2}{*}{ P. ramorum } & CBS 101553 & EU427470 & $39.5 \mathrm{~kb}$ & Y & $1.2 \mathrm{~kb}$ \\
\hline & & Pr-102 & DQ832718 & $39.3 \mathrm{~kb}$ & Y & $1.2 \mathrm{~kb}$ \\
\hline & P. sojae & P6497 & DQ832717 & $43.0 \mathrm{~kb}$ & $N$ & \\
\hline \multirow[t]{2}{*}{ Pythiaceae } & Pythium insidiosum & Pi-S & AP014838 & $55.0 \mathrm{~kb}$ & Y & $18.3 \mathrm{~kb}$ \\
\hline & P. ultimum & DAOM:BR114 & GU138662 & $59.7 \mathrm{~kb}$ & Y & $22 \mathrm{~kb}$ \\
\hline \multirow[t]{5}{*}{ Saprolegniaceae } & Achlya hypogyna & & KF226724 & $46.8 \mathrm{~kb}$ & Y & $7.97 \mathrm{~kb}$ \\
\hline & Aphanomyces astaci & AP03 & KX405004 & $49.5 \mathrm{~kb}$ & Y & $12.6 \mathrm{~kb}$ \\
\hline & A. invadans & NJM9701 & KX405005 & $49.1 \mathrm{~kb}$ & Y & $12.4 \mathrm{~kb}$ \\
\hline & Saprolegnia ferax & ATCC 36051 & AY534144 & $46.9 \mathrm{~kb}$ & Y & $8.6 \mathrm{~kb}$ \\
\hline & Thraustotheca clavata & & NC022179 & $47.4 \mathrm{~kb}$ & Y & $9.4 \mathrm{~kb}$ \\
\hline
\end{tabular}

\section{Methods}

\section{Phenotyping of isolates and DNA extraction}

Genomic DNA samples for sequencing were obtained from two isolates of $P$. effusa collected from grower fields in Monterey County, California, in 2012 and 2013. The pathotypes of these isolates were determined by inoculation onto a differential set of spinach cultivars as previously described [8]. For both isolates, leaves of plants of a single cultivar showing heavy sporulation were collected and the spores were scraped off the leaf surface into water in a $50 \mathrm{ml}$ tube as well as vortexed to remove additional sporangia. The suspension of the sporangia was transferred to a microfuge tube and spun at $21000 \mathrm{x} g$ for $1.5 \mathrm{~min}$. The resulting pellet was washed in $1 \mathrm{ml}$ of $95 \%$ ethanol, spun at 21000x $g$ for $3 \mathrm{~min}$ and the pellet frozen at $-80{ }^{\circ} \mathrm{C}$. Four hundred microliters of the Macherey-Nagel NucleoSpin Plant II kit (Düren, Germany) buffer PL1 and a single microfuge tube cap full of glass beads (Sigma G8772) was added and vortexed. The suspension was heat shocked at $65{ }^{\circ} \mathrm{C}$ with $10 \mu \mathrm{l}$ RNase A solution, followed by another high-speed vortex. A $100 \mu \mathrm{l}$ volume of chloroform was added, followed by a brief vortex and centrifugation for $5 \mathrm{~min}$ at $21000 \mathrm{~g}$. The supernatant was added to a NucleoSpin ${ }^{\bullet}$ column (Machery-Nagel NucleoSpin Plant II kit), and the manufacturer's plant DNA extraction protocol was followed.

\section{Flow cytometry}

Flow cytometry was performed on sporulating and pre-sporulating spinach leaves mixed with $1 \mathrm{~cm}^{2}$ of young leaf tissue from Oryza sativa cv. Kitaake $(2 \mathrm{C}=$ $867 \mathrm{Mb}$ ), which was sufficiently different from the genome size of $P$. effusa for use as the internal reference. The O. sativa $2 \mathrm{C}$ DNA content was determined by calibrating against nuclei from flower buds of Arabidopsis thaliana Col-0 which has a known absolute DNA content of $2 \mathrm{C}=314 \mathrm{Mb}$ [62]. Nuclei extraction and staining with propidium iodide was done using the Cystain PI absolute P kit (Sysmex, Lincolnshire, IL). Flow cytometry 
was done on a BD FACScan (Becton Dickinson, East Rutherford, NJ). For each measurement, 10,000 nuclei were assessed. Data was analyzed using FlowJo (Ashland, OR).

\section{Sequencing}

Illumina TruSeq DNA libraries were prepared and sequenced at the Center for Genome Research \& Biocomputing, Oregon State University (Corvallis, OR; http:// cgrb.oregonstate.edu/core). DNA was quantified using a Qubit HS dsDNA assay (Invitrogen, Carlsbad, CA) and sheared by sonication followed by end repair, adenylation of $3^{\prime}$ ends, and adapter ligation. Fragments were purified by excision from an agarose gel, enriched by PCR, and the library was quantified with a Qubit HS dsDNA assay (Invitrogen). Sizing of the library was done using Agilent Bioanalyzer HS-DNA chip (Agilent Technologies, Waldbronn, Germany), with final quantification by qPCR using a KAPA Library quantification kit. The median library fragment sizes were $516 \mathrm{bp}$ and $365 \mathrm{bp}$, for R13 and R14, respectively. Paired end libraries were prepared from genomic DNA of two $P$. effusa isolates (R13 and R14) and sequenced, $100 \mathrm{bp}$ paired-end on an Illumina HiSeq 2000.

\section{Assembly}

Reads were adapter and quality trimmed using BBMAP [63] and mapped to a reference containing bacterial and oomycete genomes available from NCBI, using BWA MEM, v0.7.12 [64], with flags $-\mathrm{aC}$. Paired reads which mapped to oomycete genomes or failed to map to any organism were then advanced to assembly with MaSuRCA v2.3.2 [65]. Assemblies were done, specifying a JELLYFISH [30] size of $1 \times 10^{10}$, in iterative k-mer steps of 10 , ranging from 31 to 91 , and 99 with additional assemblies performed at single step k-mer sizes flanking the highest scoring assemblies (measured on $\mathrm{N}_{50}$, assembly size, number of scaffolds $>100 \mathrm{~kb}$, total number of scaffolds, BUSCO [27] score). The top five assemblies of each isolate were then positively filtered for oomycete scaffolds against NCBI nt, and negatively filtered for mitochondrial associated scaffolds with the mitochondrial assembly (produced as described below) using BLASTn [26]. Assemblies made up of scaffolds with a top BLASTn hit against oomycete scaffolds and with a minimum scaffold size of $1 \mathrm{~kb}$, were then merged in a step-wise manner from lowest scaffold number to highest using Quickmerge [66]. Repeat libraries were generated with RepeatModeler [32], assemblies soft-masked with RepeatMasker [67] and secondary haplotypes collapsed, first with Redundans [28], then Haplomerger2 [29]. Final assembly statistics were obtained using BBMAP [63] and compared to previously assembled genomes. Completeness statistics were obtained with BUSCO.
Assemblies were aligned against one another with NUCmer [68] (-1 100), and both were independently aligned against $P$. sojae v3.0 with PROmer [68] (-1 30). These were ran and visualized as part of SyMap [31], with a minimum dot requirement of 5 and allowing merged blocks. REAPR [69] summary scores of the final and intermediate assemblies were calculated as previous [70]. REAPR summary scores of both isolates increased as post-assembly processing was performed on each isolate with the final draft assemblies presented here having the highest score (Additional files 2 and 14).

\section{Annotation}

The annotation workflow is depicted in Additional file 8 . $A b$ initio annotation was performed with SNAP [33] as part of the MAKER pipeline [34]. Initial predictions were generated with MAKER from all oomycete EST and protein data available on NCBI (est2genome $=1$, protein2genome $=1$ ) masking with the above generated repeat library. These predictions were used to produce a HMM using SNAP default settings (fathom, forge, hmm-assembler.pl) and then bootstrapped through subsequent rounds of MAKER, the first using the est2genome and protein2genome evidence for prediction, with subsequent runs turning this off to use only the SNAP $\mathrm{HMM}$ for gene prediction (est2genome $=0$, protein2genome $=0$ ). All maker runs surveyed single exon proteins with a minimum nucleotide length of 240 being considered (single_exon $=1$, single_length $=240$ ). The optimal run was identified through comparative analysis of alternative predictions, namely runs were scored for BLAST [26] hits to the Oomycete training protein sequences, \% orthology detected with the Oomycete training database as detected by OrthoFinder [71] and average e-value of Pfam [35] domains detected with InterProScan [72] with a value under $1 \mathrm{e}^{-5}$.

Additional putative effector gene models were identified from all ORFs predicted from the genome over 80 residues in length with no missing data. All ORFs were surveyed for secretion signals using SignalP v4.1 $(-\mathrm{u} 0.34)$ and independently with SignalP v3.0 (default settings) [73, 74]. ORFs were considered secreted if a positive result was obtained through either or both approaches and no trans-membrane domains were detected by SignalP v4.1. Further filtering, removing ORFs targeted to the mitochondria was performed using TargetP [75]. All ORFs predicted (i.e. regardless of signal peptide prediction) were surveyed for Crinklers (CRN) and WY repeats using hidden Markov models (HMMs) with HMMER v3.1b1 [76]. The CRN HMM was produced with hmmsearch, inputting an alignment of all labelled Phytophthora spp. CRNs from NCBI. The WY HMM was described previously [77, 78]. Predicted secreted ORFs were surveyed for 
RxLR motifs and previously described/queried degenerate [GHQ]xLR or RxL[GKQ] motifs [53, 79-82] linked with either degenerate [DE][DE][ER] motif or a WY repeat [78]. Predicted effectors were integrated into the Maker produced GFF file by identifying over-lapping gene models on the same strand with BEDTools [83]. Gene models were characterized as to whether they shared either or both start and stop codon positions.

Candidate effectors at unique genomic loci were further curated to ensure that they did not overlap or neighbor one another within $1 \mathrm{~kb}$ on the same strand. In instances when not the case, these models were manually refined, with the aid of BLAST [26], to produce multi-exonic RxLR-(EER)-WY models $[84,85]$.

The final predicted protein sequence was then generated from the GFF using GAG and putative effectors were re-identified on the entire annotation set to obtain final putative effector counts, which include those independently predicted by MAKER [34]. Conserved functional domains, including pathogenicity associated domains, of the final gene models were identified with InterProScan [72] and putative gene model names were assigned through stringent BLAST to the UniProt reference database [86]. These features were added to the NCBI table file with Annie and GAG [87, 88].

\section{Comparative genomics}

De novo identification of genes encoding pathogenicity associated domains for $P$. effusa, other downy mildew and Phytophthora species was performed by running InterProScan on all available gene models. Gene models were inferred to contain a pathogenicity associated domain if such a domain was identified by CDD, Gene3d, PANTHER, Pfam, PRINTS, ProDom, ProSitePatterns, ProSiteProfiles, SMART or SUPERFAMILY [35, 72, 89-94]. The total frequency of gene models encoding pathogenicity associated domains was calculated as the number of unique gene models identified with any of the specified domains divided by the total number of gene models surveyed. T-tests were performed on the frequency of models containing each domain and on the total frequency of pathogenicity associated gene models, segregating the results as downy mildew vs. Phytophthora and Peronospora spp., and $H$. arabidopsidis vs. Phytophthora spp. and $P$. halstedii. Chi-squared testing was performed on Pfam [35] domains detected across all seven species. In this test, a protein was counted once for each unique domain it encoded (i.e. it was not weighted if it encoded multiple of a single domain type). Expected scores were weighted for the number of proteins detected as having Pfam domains in each species. Tests were performed on all domains as $P$. effusa vs. all, Peronospora spp. vs. all, Peronospora spp. and $H$. arabidopsidis vs. all, and downy mildews vs. Phytophthora spp. A Bonferroni-adjusted $p$-value was calculated based on the number of domains tested, with Pfam domain scoring below this p-value manually investigated. Instances where an over-representation of domains in P. tabacina appeared to score a significant result were investigated, owing to the high duplication in the P. tabacina assemblies detected in this study.

Orthogroups were inferred using OrthoFinder [71]. First gene models of $P$. effusa isolates were compared in a two-way analysis. Proteins detected as absent between isolates were investigated by mapping reads of one isolate to the other isolates assembly using BWA MEM v0.7.12 [64] and calling SNPs/Indels with SAMtools mpileup v0.1.18 [95]. For comparison, the same was replicated on the two $P$. tabacina isolates. These isolates were then combined into a set for their respective species and a seven-way comparison was performed, including $H$. arabidopsidis, $P$. infestans, $P$. ramorum, $P$. sojae and $P$. halstedii. Intersects were visualized with upsetR [96] and orthology coefficients (OC) were calculated as $\mathrm{OC}=\left(\mathrm{C} / \mathrm{T}_{1}\right) \times\left(\mathrm{C} / \mathrm{T}_{2}\right)$, where $\mathrm{C}$ is the number of overlapping orthologous groups, $\mathrm{T}_{1}$ is the total number of orthologous groups identified in sample 1 and $T_{2}$ is the total number of orthologous groups identified in sample 2. Orthogroups which contained gene models encoding Pfam domains, inferred as under-represented by the Chi-squared analysis, in downy mildew assemblies were identified and analyzed for presence/absence of each species. An orthogroup was considered single copy if only $0-1$ models were present for each Phytophthora spp., $P$. halstedii and $H$. arabidopsidis and 0-2 models detected for $P$. tabacina and $P$. effusa, owing to a) two isolates being used in the analysis for both Peronospora spp. and b) the high level of duplication detected in $P$. tabacina assemblies. Finally, orthogroups containing previously identified $P$. infestans nitrogen and sulphur assimilation enzymes [45] were identified to obtain orthologs of these enzymes in downy mildew species.

\section{K-mer and read analysis}

JELLYFISH [30] 21-mer hashes for individual read files were generated and histograms were obtained to estimate the genome size of both isolates. These were plotted with R [97] to obtain the 21-mer boundaries of single copy regions of the genome. K-mer based genome size estimates were calculated by summing the results of k-mer density multiplied by its frequency. Estimates of the size of the single copy portion of the genome were produced by limiting this calculations to $\mathrm{k}$-mers with densities between the limits of the k-mer peak profiles (R13; 82-340, R14; 142-560; Additional file 13). Hashes were visually inspected through KAT density plots [36], for both $P$. effusa isolates and comparatively for two previously sequenced P. tabacina isolates [23]. Hashes generated from pairs of read files were also compared to 
assembly hashes generated from resulting isolate assemblies and visually inspected through KAT spectra-cn plots.

Heterozygosity of each isolate was estimated by calling SNPs from reads mapped back to either assembly, using SAMtools v0.1.18 mpileup [95] and calculating the number of heterozygous sites, defined as those with an allele frequency between 0.2 and 0.8 . Plotting the frequency of the alternative allele of heterozygous SNPs was performed by extracting the number of reads supporting the reference and alternative allele at each bi-allelic SNP site and obtaining the frequency of reads supporting the alternative allele and binned to the nearest hundredth. For SNP sites to be counted they had to be covered by a minimum of 50 reads. The number of SNPs per bin were summed and plotted in R [97]. Variant call files containing the SNP coordinates were intersected with genic lines of the annotated gff using BEDTools [83] to obtain SNP counts within genic regions and the allele frequency plots were produced as above. Read coverage per gene was calculated by obtaining the number of reads per genic locus, using BEDTools multicov [83] requiring the assembly file and an indexed binary alignment map file, multiplied by the read length, divided by the length of the gene. The coverage was normalized for each isolate (calculated from a BEDTools genomecov plot) and plotted in R using ggplot2 [98] applying a multiplicate bandwidth adjustment of ten.

\section{Phylogenomics}

Phylogenetics of BUSCO [27] orthologs across a panel of published, publicly available downy mildew assemblies was carried out with select, high quality Phytophthora assemblies and rooted with Pythium ultimum. BUSCO was run independently on every assembly to be surveyed and single copy orthologs from each were identified. Amino acid sequences of orthologs present in all isolates were then aligned independently using MAFFT v7.123b [99] (Additional file 15). Alignments were concatenated and RAxML v8.0.26 [100] was run with 1000 bootstraps and the PROTGAMMAAUTO substitution model. The resulting tree was rooted with $P$. ultimum and visualized in Geneious and labels were manually placed to improve legibility.

\section{Mitochondrial assembly and annotation}

Contigs from a de novo genomic assembly in CLC Genomics Workbench (v8; Qiagen, Redwood City, CA) were identified as mitochondrial due to sequence similarity with $P$. tabacina mitochondrial sequences (KT893455) by BLAST analysis. These were used as templates for further assembly with SeqMan NGen (v4.1.2, DNASTAR, Madison, WI, USA). The resulting assemblies were evaluated for uniformity and depth of coverage. Contigs were broken when gaps/low coverage or inconsistencies were observed and the set of smaller contigs reassembled using the small templated assembly option of SeqMan NGen to extend the ends of the contigs and the close gaps. ORFs were predicted and annotated with DS Gene v1.5 (Accelrys, San Diego, CA) using the universal genetic code with confirmation of gene identities using BLAST [26] analysis against mitochondrial genome sequences published for P. tabacina, Pythium, and Phytophthora spp. [23, 38, 40] tRNA coding regions were placed using tRNAscan-SE v1.3.1 [101].

\section{Additional files} Additional file 1: Flow cytometry measurements of a P. effusa isolate.
(PDF $305 \mathrm{~kb}$ )

Additional file 2: Intermediate assembly statistics and REAPR scores. (XLSX $24 \mathrm{~kb}$ )

Additional file 3: JELLYFISH histogram outputs and line plots. (7Z $171 \mathrm{~kb}$ ) Additional file 4: RepeatModeler summary of outputs. (XLSX 8 kb)

Additional file 5: Long Terminal Repeat plots. Long terminal repeats are plotted as previously described [23]. The top panel show the distribution of insertion estimates for each LTR family, the number to the right reports the number of members in that family. The bottom panel estimates the time from initial insertion of the major LTR super-families; retrotransposon LTR Gypsy (RLG) and retrotransposon LTR Copia (RLC). (JPEG 243 kb)

Additional file 6: Intermediate annotation results. Summary of maker runs bootstrapping SNAP hidden markov model. (XLSX 13 kb)

Additional file 7: Annotation pipeline. Workflow overview for the annotation of $P$. effusa. (PNG $242 \mathrm{~kb}$ )

Additional file 8: ORF annotation results. Summary of the results from $\mathrm{hmm}$ search and regular expression string searches when applied to all open reading frames of both $P$. effusa isolates. (XLSX $8 \mathrm{~kb}$ )

Additional file 9: Manual ORF curation. Image summarizing the initial ORFs predicted, manually curated exon structure, and RXLR, EER and WY locations along genomic fragments of both $P$. effusa isolates. Three were annotated in each isolate. The ORFs are not aligned. (PNG 123 kb)

Additional file 10: Significant chi-squared results. All significant Chi squared results detected, when comparing the occurrence of domains encoded by $P$. effusa, $P$. tabacina, $H$. arabidopsidis, $P$. halstedii, $P$. ramorum, $P$. sojae and $P$. infestans. (XLSX $32 \mathrm{~kb}$ )

Additional file 11: Read alignment summary of isolate specific genes. Details genes which were found to contain inter-isolate indels or SNPs which affected either the start or stop codon position of the protein. To query P.effusa proteins in GenBank, substitute PeffR13 with DD237, PeffR14 with DD238 and remove the -RA suffix. (XLSX $17 \mathrm{~kb}$ )

Additional file 12: Orthogroups unique to Peronospora spp. Details sequence names of all proteins inferred as being orthologous only between $P$. effusa and P. tabacina. To query P. effusa proteins in GenBank, substitute PeffR13 with DD237, PeffR14 with DD238 and remove the -RA suffix. (XLSX 10 kb)

Additional file 13: Orthogroup overview. Listing all the orthogroups identified and the genes they contain, which were plotted in Fig. 4. To query P. effusa proteins in GenBank, substitute PeffR13 with DD237, PeffR14 with DD238 and remove the -RA suffix. (7Z $21 \mathrm{~kb}$ )

Additional file 14: REAPR scores. Summarizing the REAPR scores reported in Additional file 1. (JPEG $89 \mathrm{~kb}$ )

Additional file 15: BUSCO alignments used to create Fig. 5. The alignments are not concatenated. Note: $7 z$ files can be accessed by downloading 7zip. This software is free and available at https://www.7zip.org/. (7Z 50 kb) 


\section{Abbreviations}

bp: Base-pair; CRN: Crinkler; H. arabidopsidis: Hyaloperonospora arabidopsidis; HMM: Hidden Markov model; IR: Inverted repeat; kb: Kilo-base-pair; LTR: Long terminal repeat; Mb: Mega-base-pair; ml: Milliliter; ORF: Open reading framw; P. cubensis: Pseudoperonospora cubensis; P. effusa: Peronospora effusa; P. farinosa: Peronospora farinosa; P. halstedii: Plasmopara halstedii; $P$. infestans: Phytophthora infestans; P. ramorum: Phytophthora ramorum; P. sojae: Phytophthora sojae; P. tabacina: Peronospora tabacina; P. ultimum: Pythium ultimum; R: Race; R13: Race 13; R14: Race 14; S. graminicola: Sclerospora graminicola; S. oleracea: Spinacia oleracea; SNPs: Single nucleotide polymorphisms; spp.: Species; $\mu$ l: Microliter

\section{Acknowledgments}

We thank Amy Anchieta (USDA ARS, Salinas) for performing the DNA extraction of $P$. effusa isolates R13 and R14, Huaqin Xu for database support, and acknowledge Oregon State University, where the sequencing of both $P$. effusa isolates was performed. This project was supported by the University of California Davis Flow Cytometry Shared Resource Laboratory with funding from the NCI P30 CA093373 (Cancer Center), and NIH NCRR C06-RR12088, S10 OD018223, S10 RR12964 and S10 RR 026825 grants and with technical assistance from Ms. Bridget McLaughlin and Mr. Jonathan Van Dyke.

\section{Funding}

The work was supported by The Novozymes Inc. Endowed Chair in Genomics to RWM.

\section{Availability of data and materials}

All sequence data is available under BioProject PRJNA453556.

\section{Authors' contributions}

KF performed the assembly, annotation, and in silico nuclear analysis as well as drafted the manuscript; SKI, SKO, BM collected and characterized the isolates and prepared sequencing libraries; LD \& SR assisted the in silico analysis; FM performed the in silico mitochondrial analysis; LB performed the flow cytometry; RM provided conceptual input and supervision as well as made major contributions to writing the manuscript. All authors contributed to writing and approved the final manuscript.

\section{Ethics approval and consent to participate}

Not applicable.

\section{Consent for publication}

Not applicable.

\section{Competing interests}

The authors declare that they have no competing interests.

\section{Publisher's Note}

Springer Nature remains neutral with regard to jurisdictional claims in published maps and institutional affiliations.

\section{Author details}

${ }^{1}$ The Genome Center, Genome and Biomedical Sciences Facility, University of California, 451 East Health Sciences Drive, Davis, CA 95616, USA. ²United States Department of Agriculture, Agricultural Research Service, Salinas, CA 93905, USA. ${ }^{3}$ UC Davis Cooperative Extension Monterey County, Salinas, CA 93901, USA. ${ }^{4}$ Departments of Plant Sciences, Molecular \& Cellular Biology, Medical Microbiology \& Immunology, University of California, Davis 95616, USA. ${ }^{5}$ Present Address: The Sainsbury Laboratory, Norwich Research Park, Norwich NR4 7UH, UK. ${ }^{6}$ Present Address: TriCal Diagnostics, Hollister, CA 95023, USA.
Received: 30 April 2018 Accepted: 31 October 2018

Published online: 29 November 2018

\section{References}

1. Thines M, Choi YJ. Evolution, diversity, and taxonomy of the Peronosporaceae, with focus on the genus Peronospora. Phytopathology. 2016:106(1):6-18.

2. McCarthy CGP, Fitzpatrick DA. Phylogenomic Reconstruction of the Oomycete Phylogeny Derived from 37 Genomes. mSphere. 2017;2(2): e00095-17.

3. Sharma R, Xia X, Cano LM, Evangelisti E, Kemen E, Judelson H, Oome S, Sambles C, van den Hoogen DJ, Kitner M, et al. Genome analyses of the sunflower pathogen Plasmopara halstedii provide insights into effector evolution in downy mildews and Phytophthora. BMC Genomics. 2015;16(1):741.

4. Ye W, Wang Y, Shen D, Li D, Pu T, Jiang Z, Zhang Z, Zheng X, Tyler BM, Wang $Y$. Sequencing of the litchi downy blight pathogen reveals it is a Phytophthora species with downy mildew-like characteristics. Mol Plant-Microbe Interact. 2016;29(7):573-83.

5. Correll JC, Bluhm BH, Feng C, Lamour K, du Toit LJ, Koike ST. Spinach: better management of downy mildew and white rust through genomics. Eur J Plant Pathol. 2011;129(2):193-205.

6. Byford W. Host specialization of Peronospora farinosa on Beta, Spinacia and Chenopodium. Trans Br Mycol Soc. 1967;50(4):603-7.

7. Klosterman SJ, Anchieta A, McRoberts N, Koike ST, Subbarao KV, Voglmayr H, Choi YJ, Thines M, Martin FN. Coupling spore traps and quantitative PCR assays for detection of the downy mildew pathogens of spinach (Peronospora effusa) and beet (P. schachtii). Phytopathology. 2014;104(12):1349-59.

8. Feng C, Correll JC, Kammeijer KE, Koike ST. Identification of new races and deviating strains of the spinach downy mildew pathogen Peronospora farinosa f. sp. spinaciae. Plant Dis. 2013;98(1):145-52.

9. Feng C, Saito K, Liu B, Manley A, Kammeijer K, Mauzey SJ, Koike S, Correll JC. New races and novel strains of the spinach downy mildew pathogen Peronospora effusa. Plant Dis. 2018;102(3):613-8.

10. Van Asch MAJ, Frinking HD. Heterothallism in Peronospora farinosa f.sp. spinaciae. Trans Br Mycol Soc. 1988:91(4):692-3.

11. Choi YJ, Hong SB, Shin HD. Re-consideration of Peronospora farinosa infecting Spinacia oleracea as distinct species, Peronospora effusa. Mycol Res. 2007;111(Pt 4):381-91.

12. Choi YJ, Klosterman SJ, Kummer V, Voglmayr H, Shin HD, Thines M. Multilocus tree and species tree approaches toward resolving a complex clade of downy mildews (Straminipila, Oomycota), including pathogens of beet and spinach. Mol Phylogenet Evol. 2015;86:24-34.

13. Choi Y-J, Thines M. (2288) Proposal to reject the name Botrytis farinosa (Peronospora farinosa) (Peronosporaceae: oomycetes). Taxon. 2014;63(3):675-6.

14. Minor T Bond J. Vegetables and Pulses Outlook. Washington DC: U.S. Department of Agriculture, Economic Research Service, Situation and Outlook, VGS-358. 2017. Retrieved from: http://usda.mannlib.cornell.edu/ usda/ers/NGS//2010s/2017NGS-04-28-2017.pdf. Accessed 8 Nov 2018.

15. Koike $S$, Cahn M, Cantwell M, Fennimore $S$, Lestrange M, Natwick E, Smith RF, Takele E. Spinach production in California. Univ Calif Agric Nat Resour Publ. 2011;7212. https://escholarship.org/uc/item/67w2p91c. Accessed 8 Nov 2018.

16. Xu C, Jiao C, Sun H, Cai X, Wang X, Ge C, Zheng Y, Liu W, Sun X, Xu Y, et al. Draft genome of spinach and transcriptome diversity of 120 Spinacia accessions. Nat Commun. 2017;8:15275.

17. Brandenberger $\mathrm{L}$, Correll J, Morelock T. Identification of and cultivar reactions to a new race (race 4 ) of Peronospora farinosa $f . s p$. spinaciae on spinach in the United States. Plant Dis. 1991;75(6):630-4.

18. Correll J. Denomination of Pfs: 17 , a new race of downy mildew in spinach. In: ANR Blogs: University of California Cooperative Extension, Division of Agriculture and Natural Resources; 2018. https://ucanr.edu/blogs/blogcore/ postdetail.cfm?postnum=26906. Accessed 8 Nov 2018.

19. Lyon R, Correll J, Feng C, Bluhm B, Shrestha S, Shi A, Lamour K. Population structure of Peronospora effusa in the southwestern United States. PLoS One. 2016;11(2):e0148385.

20. Kunjeti SG, Anchieta A, Subbarao KV, Koike ST, Klosterman SJ. Plasmolysis and vital staining reveal viable oospores of Peronospora effusa in spinach seed lots. Plant Dis. 2015;100(1):59-65. 
21. Inaba T, Morinaka T. Heterothallism in Peronospora effusa. Phytopathology. 1984;74(2):214-6.

22. Voglmayr H, Greilhuber J. Genome size determination in peronosporales (Oomycota) by Feulgen image analysis. Fungal Genet Biol. 1998;25(3):181-95.

23. Derevnina L, Chin-Wo-Reyes S, Martin F, Wood K, Froenicke L, Spring O, Michelmore R. Genome sequence and architecture of the tobacco downy mildew pathogen Peronospora tabacina. Mol Plant-Microbe Interact. 2015;28(11):1198-215.

24. Dussert Y, Gouzy J, Richart-Cervera S, Mazet ID, Delière L, Couture C, Legrand L, Piron M-C, Mestre P, Delmotte F. Draft genome sequence of Plasmopara viticola, the grapevine downy mildew pathogen. Genome Announc. 2016;4(5):e00987-16.

25. Yin L, An Y, Qu J, Li X, Zhang Y, Dry I, Wu H, Lu J. Genome sequence of Plasmopara viticola and insight into the pathogenic mechanism. Sci Rep. 2017;7:46553.

26. Altschul SF, Gish W, Miller W, Myers EW, Lipman DJ. Basic local alignment search tool. J Mol Biol. 1990:215(3):403-10.

27. Simao FA, Waterhouse RM, loannidis P, Kriventseva EV, Zdobnov EM. BUSCO: assessing genome assembly and annotation completeness with single-copy orthologs. Bioinformatics. 2015;31(19):3210-2.

28. Pryszcz LP, Gabaldon T. Redundans: an assembly pipeline for highly heterozygous genomes. Nucleic Acids Res. 2016;44(12):e113.

29. Huang S, Kang M, Xu A. HaploMerger2: rebuilding both haploid subassemblies from high-heterozygosity diploid genome assembly. Bioinformatics. 2017:33:2577-9.

30. Marçais $G$, Kingsford C. A fast, lock-free approach for efficient parallel counting of occurrences of k-mers. Bioinformatics. 2011;27(6):764-70.

31. Soderlund C, Nelson W, Shoemaker A, Paterson A. SyMAP: a system for discovering and viewing syntenic regions of FPC maps. Genome Res. 2006;16(9):1159-68.

32. Smit A, Hubley R. RepeatModeler Open-1.0.; 2008-2015.

33. Korf I. Gene finding in novel genomes. BMC Bioinformatics. 2004;5(1):59.

34. Cantarel BL, Korf I, Robb SMC, Parra G, Ross E, Moore B, Holt C, Sánchez Alvarado A, Yandell M. MAKER: an easy-to-use annotation pipeline designed for emerging model organism genomes. Genome Res. 2008;18(1):188-96.

35. Finn RD, Bateman A, Clements J, Coggill P, Eberhardt RY, Eddy SR, Heger A, Hetherington K, Holm L, Mistry J, et al. Pfam: the protein families database. Nucleic Acids Res. 2014;42(D1):D222-30.

36. Mapleson D, Garcia Accinelli G, Kettleborough G, Wright J, Clavijo BJ. KAT: a K-mer analysis toolkit to quality control NGS datasets and genome assemblies. Bioinformatics. 2017;33(4):574-6.

37. Grayburn WS, Hudspeth DS, Gane MK, Hudspeth ME. The mitochondrial genome of Saprolegnia ferax: organization, gene content and nucleotide sequence. Mycologia. 2004;96(5):981-9.

38. Levesque CA, Brouwer H, Cano L, Hamilton JP, Holt C, Huitema E, Raffaele S, Robideau GP, Thines M, Win J. Genome sequence of the necrotrophic plant pathogen Pythium ultimum reveals original pathogenicity mechanisms and effector repertoire. Genome Biol. 2010;11:R73.

39. Makkonen J, Vesterbacka A, Martin F, Jussila J, Diéguez-Uribeondo J, Kortet $\mathrm{R}$, Kokko H. Mitochondrial genomes and comparative genomics of Aphanomyces astaci and Aphanomyces invadans. Sci Rep. 2016;6:36089.

40. Martin FN, Bensasson D, Tyler BM, Boore JL. Mitochondrial genome sequences and comparative genomics of Phytophthora ramorum and $\mathrm{P}$. sojae. Curr Genet. 2007:51(5):285-96.

41. O'Brien MA, Misner I, Lane CE. Mitochondrial genome sequences and comparative genomics of Achlya hypogyna and Thraustotheca clavata. J Eukaryot Microbiol. 2014;61(2):146-54.

42. Yuan X, Feng C, Zhang Z, Zhang C. Complete mitochondrial genome of Phytophthora nicotianae and identification of molecular markers for the oomycetes. Front Microbiol. 2017:8:1484.

43. Bertier L, Leus L, D'hondt L, de Cock AWAM, Höfte M. Host adaptation and speciation through hybridization and polyploidy in Phytophthora. PLoS One. 2013;8(12):e85385.

44. Whittaker SL, Shattock RC, Shaw DS. Variation in DNA content of nuclei of Phytophthora infestans as measured by a microfluorimetric method using the fluorochrome DAPI. Mycol Res. 1991;95(5):602-10.

45. Baxter L, Tripathy S, Ishaque N, Boot N, Cabral A, Kemen E, Thines M, AhFong A, Anderson R, Badejoko W. Signatures of adaptation to obligate biotrophy in the Hyaloperonospora arabidopsidis genome. Science (New York, NY). 2010;330:1549-51.
46. Kasuga T, Bui M, Bernhardt E, Swiecki T, Aram K, Cano LM, Webber J, Brasier C, Press C, Grünwald NJ, et al. Host-induced aneuploidy and phenotypic diversification in the sudden oak death pathogen Phytophthora ramorum. BMC Genomics. 2016;17(1):385.

47. Li Y, Shen H, Zhou Q, Qian K, van der Lee T, Huang S. Changing ploidy as a strategy: the Irish potato famine pathogen shifts ploidy in relation to its sexuality. Mol Plant-Microbe Interact. 2016;30(1):45-52.

48. Spring O, Zipper R. Evidence for asexual genetic recombination in sunflower downy mildew, Plasmopara halstedii. Mycol Res. 2006;1 10(6):657-63.

49. Spring O, Zipper R. Asexual recombinants of Plasmopara halstedii Pathotypes from dual infection of sunflower. PLoS One. 2016;11(12): e0167015.

50. Yoshida K, Schuenemann VJ, Cano LM, Pais M, Mishra B, Sharma R, Lanz C, Martin FN, Kamoun S, Krause J, et al. The rise and fall of the Phytophthora infestans lineage that triggered the Irish potato famine. eLife. 2013;2:e00731.

51. Jiao $W-B$, Schneeberger $K$. The impact of third generation genomic technologies on plant genome assembly. Curr Opin Plant Biol. 2017;36:64-70.

52. Cabral A, Stassen JHM, Seidl MF, Bautor J, Parker JE, Van den Ackerveken G. Identification of Hyaloperonospora arabidopsidis transcript sequences expressed during infection reveals isolate-specific effectors. PLoS One. 2011;6(5):e19328.

53. Stassen $\mathrm{JH}$, Boer E, Vergeer PW, Andel A, Ellendorff U, Pelgrom K, Pel M, Schut J, Zonneveld O, Jeuken MJ. Specific in planta recognition of two GKLR proteins of the downy mildew Bremia lactucae revealed in a large effector screen in lettuce. Mol Plant-Microbe Interact. 2013;26(11):1259-70.

54. Sun J, Gao Z, Zhang X, Zou X, Cao L, Wang J. Transcriptome analysis of Phytophthora litchii reveals pathogenicity arsenals and confirms taxonomic status. PLoS One. 2017;12(6):e0178245.

55. Ah-Fong AMV, Kim KS, Judelson HS. RNA-seq of life stages of the oomycete Phytophthora infestans reveals dynamic changes in metabolic, signal transduction, and pathogenesis genes and a major role for calcium signaling in development. BMC Genomics. 2017;18(1):198.

56. Judelson HS. Dynamics and innovations within oomycete genomes: insights into biology, pathology, and evolution. Eukaryot Cell. 2012;11(11):1304-12.

57. Lee S-J, Rose JKC. Mediation of the transition from biotrophy to necrotrophy in hemibiotrophic plant pathogens by secreted effector proteins. Plant Signal Behav. 2010;5(6):769-72.

58. Catal M, King L, Tumbalam P, Wiriyajitsomboon P, Kirk WW, Adams GC. Heterokaryotic nuclear conditions and a heterogeneous nuclear population are observed by flow cytometry in Phytophthora infestans. Cytometry A 2010;77(8):769-75.

59. Hudspeth ME, Shumard DS, Bradford CJ, Grossman LI. Organization of Achlya mtDNA: a population with two orientations and a large inverted repeat containing the rRNA genes. Proc Natl Acad Sci U S A. 1983;80(1):142-6.

60. McNabb SA, Boyd DA, Belkhiri A, Dick MW, Klassen GR. An inverted repeat comprises more than three-quarters of the mitochondrial genome in two species of Pythiumm. Curr Genet. 1987;12(3):205-8.

61. Martin FN. Mitochondrial haplotype determination in the oomycete plant pathogen Phytophthora ramorum. Curr Genet. 2008;54(1):23-34.

62. Bennett MD, Leitch IJ, Price $H J$, Johnston JS. Comparisons with Caenorhabditis ( 100 Mb) and drosophila ( 175 Mb) using flow cytometry show genome size in Arabidopsis to be $\sim 157 \mathrm{Mb}$ and thus $\sim 25 \%$ larger than the Arabidopsis genome initiative estimate of $\sim 125 \mathrm{Mb}$. Ann Bot. 2003;91(5):547-57

63. Bushnell B. BBMap short read aligner. Berkeley: University of California; 2016. https://sourceforge.net/projects/bbmap. Accessed 8 Nov 2018.

64. Li H. Aligning sequence reads, clone sequences and assembly contigs with BWA-MEM: arXiv preprint arXiv:1303.3997; 2013.

65. Zimin AV, Marçais G, Puiu D, Roberts M, Salzberg SL, Yorke JA. The MaSuRCA genome assembler. Bioinformatics. 2013;29(21):2669-77.

66. Chakraborty M, Baldwin-Brown JG, Long AD, Emerson JJ. Contiguous and accurate de novo assembly of metazoan genomes with modest long read coverage. Nucleic Acids Res. 2016;44(19):e147.

67. Smit A, Hubley R, Green P. RepeatMasker open-4.0.; 2013-2015.

68. Delcher AL, Kasif S, Fleischmann RD, Peterson J, White O, Salzberg SL. Alignment of whole genomes. Nucleic Acids Res. 1999;27(11):2369-76.

69. Hunt M, Kikuchi T, Sanders M, Newbold C, Berriman M, Otto TD. REAPR: a universal tool for genome assembly evaluation. Genome Biol. 2013;14(5):R47.

70. Bradnam KR, Fass JN, Alexandrov A, Baranay P, Bechner M, Birol I, Boisvert S, Chapman JA, Chapuis G, Chikhi R, et al. Assemblathon 2: evaluating de 
novo methods of genome assembly in three vertebrate species GigaScience. 2013;2(1):10

71. Emms DM, Kelly S. OrthoFinder: solving fundamental biases in whole genome comparisons dramatically improves orthogroup inference accuracy. Genome Biol. 2015;16(1):157.

72. Jones $\mathrm{P}$, Binns D, Chang H-Y, Fraser M, Li W, McAnulla C, McWilliam H, Maslen J, Mitchell A, Nuka G, et al. InterProScan 5: genome-scale protein function classification. Bioinformatics. 2014;30(9):1236-40.

73. Bendtsen JD, Nielsen H, von Heijne G, Brunak S. Improved prediction of signal peptides: SignalP 3.0. J Mol Biol. 2004;340(4):783-95.

74. Petersen TN, Brunak S, von Heijne G, Nielsen H. SignalP 4.0: discriminating signal peptides from transmembrane regions. Nat Methods. 2011;8(10):785-6.

75. Emanuelsson O, Nielsen H, Brunak S, von Heijne G. Predicting subcellular localization of proteins based on their $\mathrm{N}$-terminal amino acid sequence. J Mol Biol. 2000:300(4):1005-16

76. Finn RD, Clements J, Eddy SR. HMMER web server: interactive sequence similarity searching. Nucleic Acids Res. 2011:39(Web Server issue):W29-37.

77. Boutemy LS, King SRF, Win J, Hughes RK, Clarke TA, Blumenschein TMA, Kamoun S, Banfield MJ. Structures of RXLR Effector Proteins. J Biol Chem. 2011;286(41):35834-842.

78. Win J, Krasileva KV, Kamoun S, Shirasu K, Staskawicz BJ, Banfield MJ. Sequence divergent RXLR effectors share a structural fold conserved across plant pathogenic oomycete species. PLoS Pathog. 2012:8(1):e1002400.

79. Fawke S, Doumane M, Schornack S. Oomycete interactions with plants: infection strategies and resistance principles. Microbiol Mol Biol Rev. 2015;79(3):263-80

80. Mestre P, Carrere S, Gouzy J, Piron MC, Tourvieille de Labrouhe D, Vincourt P, Delmotte F, Godiard L. Comparative analysis of expressed CRN and RXLR effectors from two Plasmopara species causing grapevine and sunflower downy mildew. Plant Pathol. 2016;65(5):767-81.

81. Stassen JHM. Identification and functional analysis of downy mildew effectors in lettuce and Arabidopsis; 2012.

82. Tian M, Win J, Savory E, Burkhardt A, Held M, Brandizzi F, Day B. 454 genome sequencing of Pseudoperonospora cubensis reveals effector proteins with a QXLR translocation motif. Mol Plant-Microbe Interact. 2011;24(5):543-53.

83. Quinlan AR. BEDTools: the Swiss-army tool for genome feature analysis. Curr Protoc Bioinformatics. 2014;47:11.12.11-34 editoral board, Andreas D Baxevanis [et al].

84. Burkhardt A, Buchanan A, Cumbie JS, Savory EA, Chang JH, Day B Alternative splicing in the obligate biotrophic oomycete pathogen Pseudoperonospora cubensis. Mol Plant-Microbe Interact. 2015;28(3): 298-309.

85. Savory EA, Zou C, Adhikari BN, Hamilton JP, Buell CR, Shiu S-H, Day B. Alternative splicing of a multi-drug transporter from Pseudoperonospora cubensis generates an RXLR effector protein that elicits a rapid cell death. PLoS One. 2012;7(4):e34701.

86. The UniProt Consortium. UniProt: the universal protein knowledgebase. Nucleic Acids Res. 2017:45(D1):D158-69.

87. GAG: the Genome Annotation Generator (Version 1.0) [Software] [http:// genomeannotation.github.io/GAG]. Accessed 8 Nov 2018

88. Annie: the ANNotation Information Extractor (Version 1.0) [Software] [http:// genomeannotation.github.io/annie]. Accessed 8 Nov 2018.

89. Bru C, Courcelle E, Carrere S, Beausse Y, Dalmar S, Kahn D. The ProDom database of protein domain families: more emphasis on 3D. Nucleic Acids Res. 2005;33(Database issue):D212-5.

90. Kasuya A, Thornton JM. Three-dimensional structure analysis of PROSITE patterns. J Mol Biol. 1999;286(5):1673-91.

91. Lees J, Yeats C, Perkins J, Sillitoe I, Rentzsch R, Dessailly BH, Orengo C. Gene3D: a domain-based resource for comparative genomics, functional annotation and protein network analysis. Nucleic Acids Res. 2012; 40(Database issue):D465-71.

92. Letunic I, Bork P. "20 years of the SMART protein domain annotation resource." Nucleic Acids Res. 2017:46(D1):D493-6.

93. Marchler-Bauer A, Bo Y, Han L, He J, Lanczycki CJ, Lu S, Chitsaz F, Derbyshire MK, Geer RC, Gonzales NR, et al. CDD/SPARCLE: functional classification of proteins via subfamily domain architectures. Nucleic Acids Res. 2017:45(Database issue):D200-3.

94. Thomas PD, Kejariwal A, Campbell MJ, Mi H, Diemer K, Guo N, Ladunga I, Ulitsky-Lazareva B, Muruganujan A, Rabkin S, et al. PANTHER: a browsable database of gene products organized by biological function, using curated protein family and subfamily classification. Nucleic Acids Res. 2003;31(1): $334-41$

95. Li H, Handsaker B, Wysoker A, Fennell T, Ruan J, Homer N, Marth G, Abecasis $G$, Durbin R. The sequence alignment/map format and SAMtools. Bioinformatics. 2009;25(16):2078-9.

96. Conway JR, Lex A, Gehlenborg N. "UpSetR: an R package for the visualization of intersecting sets and their properties." Bioinformatics. 2017; 33(18):2938-40.

97. R Development Core Team. R: a language and environment for statistical computing. Vienna: R Foundation for Statistical Computing; 2012.

98. R Core Team. R: a language and environment for statistical computing. $R$ Foundation for Statistical Computing, Vienna, Austria. 2013.

99. Katoh K, Standley DM. MAFFT multiple sequence alignment software version 7: improvements in performance and usability. Mol Biol Evol. 2013:30(4):772-80

100. Stamatakis A. RAxML version 8: a tool for phylogenetic analysis and postanalysis of large phylogenies. Bioinformatics. 2014;30(9):1312-3.

101. Lowe TM, Eddy SR. tRNAscan-SE: a program for improved detection of transfer RNA genes in genomic sequence. Nucleic Acids Res. 1997;25(5):955-64

102. Nayaka, S. Chandra, et al. Draft genome sequence of Sclerospora graminicola, the pearl millet downy mildew pathogen. Biotechnology reports. 2017;16:18-20

103. Haas BJ, Kamoun S, Zody MC, Jiang RHY, Handsaker RE, Cano LM, Grabherr M, Kodira CD, Raffaele S, Torto-Alalibo T, et al. Genome sequence and analysis of the Irish potato famine pathogen Phytophthora infestans. Nature. 2009:461 (7262):393-8.

104. Tyler BM, Tripathy $S$, Zhang $X$, Dehal $P$, Jiang RH, Aerts A, Arredondo FD, Baxter L, Bensasson D, Beynon JL. Phytophthora genome sequences uncover evolutionary origins and mechanisms of pathogenesis. Science (New York, NY). 2006;313:1261-6.

\section{Ready to submit your research? Choose BMC and benefit from:}

- fast, convenient online submission

- thorough peer review by experienced researchers in your field

- rapid publication on acceptance

- support for research data, including large and complex data types

- gold Open Access which fosters wider collaboration and increased citations

- maximum visibility for your research: over $100 \mathrm{M}$ website views per year

At $\mathrm{BMC}$, research is always in progress.

Learn more biomedcentral.com/submissions 